\title{
Analytic SU(N) Skyrmions at finite baryon density
}

\author{
Pedro D. Alvarez $\odot,{ }^{1, *}$ Sergio L. Cacciatori $\odot,{ }^{2,3, \dagger}$ Fabrizio Canfora, ${ }^{4, \ddagger}$ and Bianca L. Cerchiai ${ }^{5,6,7,8, \S}$ \\ ${ }^{1}$ Departamento de Fisica, Universidad de Antofagasta, Aptdo 02800, Chile \\ ${ }^{2}$ Dipartimento di Scienza e Alta Tecnologia, Università dell'Insubria, via Valleggio 11, \\ Como, Italia Milan 22100, Italia \\ ${ }^{3}$ INFN Sezione di Milano, via Celoria 16, 20133 Milan, Italy \\ ${ }^{4}$ Centro de Estudios Científicos (CECS), Casilla 1469, Valdivia, Chile \\ ${ }^{5}$ Museo Storico della Fisica e Centro Studi e Ricerche "Enrico Fermi", \\ Piazza del Viminale 1, 00184 Roma, Italy \\ ${ }^{6}$ Politecnico di Torino, Dip. Department of Applied Science and Technology, \\ Corso Duca degli Abruzzi 24, 10129 Torino, Italy \\ ${ }^{7}$ Istituto Nazionale di Fisica Nucleare (INFN) Sezione diTorino, 10121 Torino, Italy \\ ${ }^{8}$ Arnold-Regge Center, via P. Giuria 1, 10125 Torino, Italy
}

(Received 22 December 2019; revised manuscript received 1 March 2020; accepted 21 May 2020; published 12 June 2020)

\begin{abstract}
We construct analytic $(3+1)$-dimensional Skyrmions living at finite baryon density in the $S U(N)$ Skyrme model that are not trivial embeddings of $S U(2)$ into $S U(N)$. We use Euler angle decomposition for arbitrary $N$ and the generalized hedgehog ansatz at finite baryon density. The skyrmions of high topological charge that we find represent smooth baryonic layers whose properties can be computed explicitly. In particular, we determine the energy-to-baryon charge ratio for any $N$ showing the smoothness of the large$N$ limit. The closeness to the BPS bound of these configurations can also be analyzed. The energy density profiles of these finite density skyrmions have a "lasagna-like" shape, in agreement with recent experimental findings. The shear modulus can be precisely estimated as well, and our analytical result is close to recent numerical studies in the literature.
\end{abstract}

DOI: 10.1103/PhysRevD.101.125011

\section{INTRODUCTION}

The characterization of the phase diagram of the lowenergy limit of QCD at finite baryon density and low temperatures has motivated intense research in the last two decades (see Ref. [1] and references therein). Analytic models are scarce, and new exact results are hard to obtain. A well-known example is the $(3+1)$-dimensional NambuJona-Lasinio (NJL) model, which shares some of the analytical difficulties of the low-energy limit of QCD (see Ref. [2] for a review). Together with the uselessness of perturbation theory at low energy, this means that the complicated phase diagram of low-energy QCD cannot be easily analyzed with the available analytic techniques (see Refs. [3-5] and references therein).

\footnotetext{
*pd.alvarez.n@gmail.com

sergio.cacciatori@uninsubria.it

*anfora@cecs.cl

§bianca.cerchiai@polito.it
}

Published by the American Physical Society under the terms of the Creative Commons Attribution 4.0 International license. Further distribution of this work must maintain attribution to the author(s) and the published article's title, journal citation, and DOI. Funded by SCOAP.
A remarkable feature of low-energy QCD at finite baryon density is that at low temperatures, very complex structures appear. When the baryon density is increased, a phase that is commonly defined as "nuclear pasta" appears. In Refs. [6-13], the presence of "baryonic layers" was disclosed, which will be the main focus of the present paper. Such a name arises from the fact that most of the baryonic charge and energy density are concentrated within lasagna-shaped regions in three dimensions. ${ }^{1}$ Many physical properties of these configurations are currently under investigation, such as the elasticity of nuclear pasta and its transport properties [10-13]. The high topological charge of nuclear pasta makes it hard to study analytically.

As powerful numerical techniques are available to analyze these configurations (see, for instance, Refs. [10-13] and references therein), why should one insist on finding analytic solutions? There are many reasons to strive for analytic solutions even when numerical techniques are available. First, it could be enough to note all the fundamental concepts that we have understood thanks to the availability of the Kerr solutions in general relativity and of the non-Abelian monopoles and instantons in Yang-Mills-Higgs theory. Second, as

\footnotetext{
${ }^{1}$ The phases nuclear spaghetti and nuclear gnocchi also appear in the literature; see the references quoted above.
} 
in the present case, analytic solutions can disclose relevant physical properties of very complex structures which are difficult to analyze even numerically.

Until recently, these types of nonhomogeneous condensates in the low-energy limit of QCD in $(3+1)$ dimensions could not be properly understood analytically. A further problem is that, computationally, the large- $N_{f}$ and large- $N_{c}$ limits must be addressed carefully $[14,15]$. One of the goals of the present paper is to shed light on the large- $N_{f}$ behavior of these complex structures.

A simplified version of the low-energy limit of QCD that encodes many relevant features is the $(1+1)$-dimensional version of the NJL model, also known as the chiral GrossNeveu model [16-19]. Such a model possesses a crystalline phase at low temperature and finite baryon density [20-23]. These results suggest that ordered structures must also appear in the low-energy limit of QCD. At leading order in the 't Hooft expansions [24-26], the low-energy limit of QCD is described by the Skyrme theory [27] (see Refs. [28,29] for reviews). Despite the bosonic nature of the skyrmion field $U$, its solitons represent baryons (see Refs. [26,30-33]).

Here, we analyze the appearance of complex structures at finite baryon density in the $S U(N)$ Skyrme model in $(3+1)$ dimensions. We focus on the analytic computations of relevant physical properties, such as the energy density, the energy per baryon, and the shear modulus of nuclearlasagna-like structures living at finite density. ${ }^{2}$ We compute their corresponding scaling with $N$.

We combine the use of Euler angles for $S U(N)$ developed in Refs. [39-41] together with the use of nonspherical hedgehog ansatz introduced in Refs. [42-50].

\section{SKYRME ACTION}

The action of the Skyrme model in four dimensions is

$$
S=\frac{K}{4} \int d^{4} x \sqrt{-g} \operatorname{tr}\left(R^{\mu} R_{\mu}+\frac{\lambda}{8} F_{\mu \nu} F^{\mu \nu}\right),
$$

where $R_{\mu}=U^{-1} \partial_{\mu} U=R_{\mu}^{j} t_{j}$ with $U \in S U(N), t_{i}$ the $S U(N)$ generators, $K$ and $\lambda$ the Skyrme couplings, $g$ the metric determinant, ${ }^{3}$ and $F_{\mu \nu}=\left[R_{\mu}, R_{\nu}\right]$. The field equations are

$$
\nabla^{\mu}\left(R_{\mu}+\frac{\lambda}{4}\left[R^{\nu}, F_{\mu \nu}\right]\right)=0
$$

We construct topologically nontrivial solutions at finite baryon density. Our main goal is to determine the scaling with $N$ of relevant physical quantities. As we want to

\footnotetext{
${ }^{2}$ Pioneering works on the Skyrme model at finite density are Refs. [34-38] and references therein.

${ }^{3}$ We remind the reader that the $N$ of the $S U(N)$ of the Skyrme model corresponds to $\mathbf{N}_{f}$.
}

analyze skyrmions of high topological charge living in flat spaces at finite baryon density, we consider the following metric:

$$
d s^{2}=-d t^{2}+L_{r}^{2} d r^{2}+L_{\gamma}^{2} d \gamma^{2}+L_{\varphi}^{2} d \varphi^{2},
$$

while the range of coordinates is

$$
0 \leq r \leq 2 \pi, \quad 0 \leq \gamma \leq 2 \pi, \quad 0 \leq \varphi \leq 2 \pi,
$$

with the caveat that, despite the chosen values, they are not periodic. The parameters $L_{r}, L_{\gamma}$, and $L_{\varphi}$ represent the size of the box within which the skyrmion is confined.

\section{A. Quantities of high physical interest}

First, the main goal of the paper is to compute the energy per baryon and its large- $N$ behavior. Therefore, only solutions with nonvanishing baryon charge have been considered. The usual definition of baryon charge in the Skyrme model (see Refs. [26,27,31,32]) is

$$
\begin{gathered}
W=B=\frac{1}{24 \pi^{2}} \int_{\{t=\text { const }\}} \rho_{B}, \\
\rho_{B}=\epsilon^{i j k} \operatorname{tr}\left(U^{-1} \partial_{i} U\right)\left(U^{-1} \partial_{j} U\right)\left(U^{-1} \partial_{k} U\right),
\end{gathered}
$$

so a necessary condition in order to have nontrivial topological charge is

$$
\rho_{B} \neq 0 \text {. }
$$

From the geometrical point of view, the above condition can be interpreted as saying that the skyrmion "fills a threedimensional spatial volume," at least locally. On the other hand, such a condition is not sufficient, in general. One also has to require that the spatial integral of $\rho_{B}$ be a nonvanishing integer:

$$
\frac{1}{24 \pi^{2}} \int_{\{t=\text { const }\}} \rho_{B} \in \mathbb{Z} .
$$

Usually, this second requirement allows us to fix some of the parameters and integration constants of the ansatz, as we will see in the following. However, there are more global conditions to be satisfied, as will be explained below. Hence, in the following we will only consider solutions satisfying both the condition in Eq. (2.7) and the one in Eq. (2.8).

Second, the energy density (the $0-0$ component of the energy-momentum tensor) reads

$$
\begin{aligned}
T_{00}= & -\frac{K}{2} \operatorname{tr}\left[R_{0} R_{0}-\frac{1}{2} g_{00} R^{\alpha} R_{\alpha}\right. \\
& \left.+\frac{\lambda}{4}\left(g^{\alpha \beta} F_{0 \alpha} F_{0 \beta}-\frac{g_{00}}{4} F_{\sigma \rho} F^{\sigma \rho}\right)\right],
\end{aligned}
$$


where $F_{\mu \nu}=\left[R_{\mu}, R_{\nu}\right]$. Thus, the total energy $E$ of the skyrmion is the spatial integral of the above quantity,

$$
E=\int_{\{t=\text { const }\}} \sqrt{-g} T_{00} .
$$

We define a skyrmion $U$ to be static if its energy density defined above is static. In other words, a skyrmion is static if it corresponds to a static distribution of energy density. It is worth noting that this definition is more general than the naive definition of a static skyrmion as a static $S U(N)$ valued configuration $U$ which does not depend on time. In particular, an elegant approach to avoid Derrick's famous no-go theorem on the existence of solitons corresponds to searching for a time-periodic ansatz such that the energy density of the configuration is still static, as it happens for boson stars [51] [in the simpler case of $U(1)$-charged scalar field; see Ref. [52] and references therein]. The ansatz to be defined in the next sections have exactly this property. Moreover, unlike what happens for the usual boson star ansatz for $U(1)$-charged scalar fields, the present ansatz for $S U(N)$-valued scalar fields also possesses a nontrivial topological charge. Thus, we are interested in solutions in which the energy density has nontrivial local maxima, which could be identified with the position of the skyrmions.

Given a solution of $S U(N)$ with baryonic charge $B$ and energy $E$ living in the metric (2.3) we have already mentioned, it is very interesting to analyze the following quantity [which is nothing but the energy per baryon of the configuration $g(N, a)$ ]:

$$
\frac{E}{B} \stackrel{\text { def }}{=} g(N, a),
$$

where $a$ is any set of integration constants which characterize the given solution. It is especially interesting to understand the behavior of $g(N, a)$ defined above when $N$ is large (the 't Hooft limit). Here and in the following, we will call the function $g(N, a)$ the " $g$-factor." The very deep question is whether or not, in the given family of solutions one is considering, one can define

$$
g^{*}(a)=\lim _{N \rightarrow \infty} g(N, a)
$$

and if this limit is well defined. In particular, one might like to find whether or not "the closeness to the BPS bound" improves when $N$ is large. Indeed, it is worth noting that in the $S U(2)$ case, all the known solutions with nonvanishing topological charge exceed the bound by at least $20 \%$. Hence, one might like to find whether, in the 't Hooft limit, the "closeness of skyrmions to the BPS bound" is finite or whether it grows without bound. This issue is deeply related to the so-called Veneziano limit [25], which is a variant of the 't Hooft limit in which the flavor number $N_{f}$ also goes to infinity in such a way that $N_{c} / N_{f}$ stays finite. The Veneziano limit allows us to take into account the effects of quarks while keeping the advantages of the 't Hooft topological expansion. Since, to arrive at the Skyrme model as an effective low-energy limit of QCD, $N_{c}$ must already be large, the large- $N$ limit that we are considering here [in which $N$ is the one from the $S U(N)$ Skyrme model] can be considered as a sort of Veneziano limit applied to the Skyrme model itself. The fact that such a limit is smooth is a very nontrivial result which would be very difficult to prove directly in the QCD Lagrangian.

The above discussion clearly shows that in order to declare a solution of the Skyrme field equations as "physically interesting," two criteria must be satisfied:

(1) The topological charge of the solution must be nonvanishing.

(2) The energy density $T_{00}$ as a function of the coordinates must have an interesting pattern.

\section{LOCAL SOLUTIONS}

Using the Euler angles for $S U(N)$ determined in Refs. [39,40] together with the ansatz for nonspherical skyrmions living at finite baryon density in Refs. [42-50], one arrives at the following ansatz for the $S U(N)$ skyrmion:

$$
\begin{gathered}
U[t, r, \varphi, \gamma]=e^{\Phi k} e^{h(r)} e^{m \gamma k}, \\
\Phi=\frac{t}{L_{\varphi}}-\varphi,
\end{gathered}
$$

with a suitable choice of $k$ in $\mathfrak{s} \mathfrak{t}(N)$ and $h(r)$ in the Cartan subalgebra $H$ to be specified below, $m$ a nonvanishing integer number, and where we recall that the metric is given by Eq. (2.3). When necessary to expand with respect to the basis of $\mathfrak{g} \mathfrak{t}(N)$, we also write

$$
h(r)=y_{1}(r) J_{1}+\cdots+y_{N-1}(r) J_{N-1},
$$

with (see Appendix A)

$$
J_{k}=i\left(E_{k, k}-E_{k+1, k+1}\right), \quad k=1, \ldots, N-1 .
$$

In general, we use the simplifying notations

$$
h^{\prime}=\frac{d}{d r} h(r), \quad h^{\prime \prime}=\frac{d^{2}}{d r^{2}} h(r) .
$$

As for $k$, for $c_{j}$ arbitrary complex numbers, forming the components of the vector $\underline{c} \in \mathbb{C}^{N-1}$, we choose

$$
k \equiv k_{\underline{c}}=\sum_{j=1}^{N-1}\left(c_{j} \lambda_{j}-c_{j}^{*} \lambda_{j}^{\dagger}\right),
$$


$\lambda_{j} \equiv \lambda_{\alpha_{j}}$ being the eigenmatrices of the simple roots (Appendix A). We get the following proposition.

Proposition 1: From the ansatz (3.1), (3.2), and (2.3), the equations of motion reduce to

$$
h^{\prime \prime}=\frac{\lambda m^{2}}{4 L_{\gamma}^{2}}\left(\left[k,\left[k, h^{\prime \prime}\right]\right]-\left[k,\left[h^{\prime},\left[h^{\prime}, k\right]\right]\right]\right),
$$

where the prime indicates derivation with respect to $r$.

The proof is given in Appendix B. Exploiting Eqs. (3.3) and (3.6), we can further simplify the equations of motion, which can be put in the following form:

$$
h^{\prime \prime}+\frac{\lambda m^{2}}{2 L_{\gamma}^{2}} \sum_{j=1}^{N-1} \alpha_{j}\left(h^{\prime \prime}\right)\left|c_{j}\right|^{2} J_{j}=0
$$

$$
\sum_{j<k}\left(\alpha_{j}\left(h^{\prime}\right)^{2}-\alpha_{k}\left(h^{\prime}\right)^{2}-i\left(\alpha_{j}\left(h^{\prime \prime}\right)-\alpha_{k}\left(h^{\prime \prime}\right)\right)\right) c_{j} c_{k}\left[\lambda_{j}, \lambda_{k}\right]-\text { H.c. }=0,
$$

Now, we use general properties of simple roots. Since $\lambda_{j}$ are eigenmatrices relative to simple roots, we get that $\left[\lambda_{j}, \lambda_{k}\right]=0$ or that it is an eigenmatrix relative to a positive root. ${ }^{4}$ Similar considerations follow for $\lambda_{j}^{\dagger}$ with respect to negative roots. It follows that none of these terms can lie in $H$; thus, projecting Eq. (3.10) on $H$, we get ( $h^{\prime \prime}$ belongs in $H$ by definition) Eq. (3.8), while projecting on the complement, we get Eq. (3.9). These equations could be expressed even more explicitly in components, by exploiting Eq. (3.3) and using that $\alpha_{j}\left(J_{k}\right)=C_{A_{N-1}, k}$ are the components of the Cartan matrix of $S U(N)$, as defined in Appendix A 2, so that $\alpha_{j}\left(h^{(n)}\right)=2 y_{j}^{(n)}-y_{j+1}^{(n)}-y_{j-1}^{(n)}$. However, such an explicit expression is not necessary in order to get the general solution.

\section{A. Explicit solutions}

Now, we want to find all the solutions of Eqs. (3.8) and (3.9). To this end, we make use of some technical facts explained in Appendix A 2. Let us first consider Eq. (3.9). Using Eq. (A16) it becomes

$$
\sum_{j=1}^{N-2}\left(\alpha_{j}\left(h^{\prime}\right)^{2}-\alpha_{j+1}\left(h^{\prime}\right)^{2}-i\left(\alpha_{j}\left(h^{\prime \prime}\right)-\alpha_{j+1}\left(h^{\prime \prime}\right)\right)\right) c_{j} c_{j+1} E_{j, j+2}-\text { H.c. }=0 .
$$

We assume $\underline{c}$ to be generic, meaning that all the $c_{j}$ are nonzero. Since $E_{j, j+2}$, including their conjugates, are all linearly independent, this gives

$$
\begin{aligned}
& \alpha_{j}\left(h^{\prime}\right)^{2}-\alpha_{j+1}\left(h^{\prime}\right)^{2}-i\left(\alpha_{j}\left(h^{\prime \prime}\right)-\alpha_{j+1}\left(h^{\prime \prime}\right)\right)=0, \\
& j=1, \ldots, N-2 .
\end{aligned}
$$

Since $\alpha_{j}$ are real valued, we also get

$$
\begin{aligned}
\alpha_{j}\left(h^{\prime \prime}\right) & =\alpha_{j+1}\left(h^{\prime \prime}\right), \quad \alpha_{j}\left(h^{\prime}\right)^{2}-\alpha_{j+1}\left(h^{\prime}\right)^{2}=0, \\
j & =1, \ldots, N-2 .
\end{aligned}
$$

\footnotetext{
${ }^{4}$ That is, a linear combination of simple roots with nonnegative integer coefficients.
}

The first two equations give

$$
\alpha_{j}\left(h^{\prime \prime}\right)=\alpha_{1}\left(h^{\prime \prime}\right), \quad j=2, \ldots, N-1 .
$$

We have two possibilities: $h^{\prime \prime}=0$ or not. We now show that the second case leads to a contradiction. First, notice that if $h^{\prime \prime} \neq 0$ then it must be $\alpha_{j}\left(h^{\prime \prime}\right) \neq 0$ for at least one $j$ (since the $\alpha_{j}$ are linearly independent) so that all $\alpha_{j}\left(h^{\prime \prime}\right)$ are equal and different from zero. From the second equation of (3.12) we have that there must exist signs $\varepsilon_{j}$ such that

$$
\alpha_{j}\left(h^{\prime}\right)=\varepsilon_{j} \alpha_{1}\left(h^{\prime}\right), \quad j=2, \ldots, N-1 .
$$

Deriving it with respect to $r$ must give (3.13); thus, $\varepsilon_{j}=1$ for all $j$, and we are left with the linear system of equations 


$$
\alpha_{j}\left(h^{\prime}\right)=\alpha_{1}\left(h^{\prime}\right), \quad j=2, \ldots, N-1 .
$$

Since the $\alpha_{j}$ are linearly independent (of rank $N-1$ ) this is a set of $N-2$ linearly independent equations for $h^{\prime} \in H$. Since $H$ is $N-1$ dimensional, the space of solutions is one dimensional, and its general solution is

$$
h^{\prime}(r)=f(r) v,
$$

where $f$ is an arbitrary function and $v \in H$ is the unique matrix satisfying $\alpha_{j}(v)=1$ for all $j$ (which we will compute later; for now it is sufficient to know it exists). We now replace this solution in Eq. (3.8). We immediately get

$$
f^{\prime}(r)\left(v+\frac{\lambda m^{2}}{2 L_{\gamma}^{2}} \sum_{j=1}^{N-1}\left|c_{j}\right|^{2} J_{j}\right)=0 .
$$

Since we have assumed $h^{\prime \prime} \neq 0$, we have $f^{\prime} \neq 0$ and, therefore,

$$
v=-\frac{\lambda m^{2}}{2 L_{\gamma}^{2}} \sum_{j=1}^{N-1}\left|c_{j}\right|^{2} J_{j}
$$

After applying $\alpha_{k}$ to this equality, using that $\alpha_{k}(v)=1$ and noticing that $\alpha_{k}\left(J_{j}\right)=C_{A_{N-1} k, j}$ are the components of the Cartan matrix, we get

$$
1=-\frac{\lambda m^{2}}{2 L_{\gamma}^{2}} \sum_{j=1}^{N-1} C_{A_{N-1} k, j}\left|c_{j}\right|^{2}, \quad j=1, \ldots, N-1 .
$$

This relation can be inverted easily: If we consider 1 at varying $j$ to be the components of a vector in $\mathbb{R}^{N-1}$, we can apply the inverse Cartan matrix to both members, thus getting

$$
\left|c_{j}\right|^{2}=-\frac{2 L_{\gamma}^{2}}{\lambda m^{2}} \sum_{k=1}^{N-1} C_{A_{N-1} k, j}^{-1} .
$$

Since $\lambda$ is positive and the same is true for the elements of the inverse Cartan matrix (A15), we see that this leads to a contradiction. Therefore, the only possibility is that $f^{\prime}(r)=0$, which is equivalent to $h^{\prime \prime}(r)=0$.

Hence, we proceed in investigating the first possibility, $h^{\prime \prime}=0$. In this case Eq. (3.8) is automatically satisfied, and Eq. (3.9) reduces to Eq. (3.14). Its solution is

$$
h^{\prime}(r)=a v
$$

where $a$ is a constant and $v \in H$ is the unique matrix solving $\alpha_{j}(v)=\varepsilon_{j}, j=1, \ldots, N-1$ where $\varepsilon_{j} \in\{0,1\}$ (and $\varepsilon_{1}=1$ ). Since $\varepsilon_{1}$ is fixed, this gives a $2^{N-2}$ solution for every choice of $c_{j}$ in $k$. As we will see in the explicit example of $S U(4)$, however, not all of these are really distinct solutions. There is a convenient way to express $v$ explicitly. Indeed, let us write $h=a r v_{\varepsilon}$, where $a$ is a constant and $v_{\varepsilon} \in H$ is a matrix,

$$
v_{\varepsilon}=\operatorname{diag}\left(v_{1}, \ldots, v_{N}\right)
$$

such that $\alpha_{i}\left(v_{\varepsilon}\right)=\varepsilon_{i}, \varepsilon_{i}= \pm 1, i=1, \ldots, N-1$ and of course $\sum_{i=1}^{N} v_{i}=0$. These equations are easily solved by writing $v=\sum_{j=1}^{n-1} w_{j} J_{j}$ so that the equations are

$$
\varepsilon_{k}=\sum_{j=1}^{N-1} C_{A_{N-1} k, j} w_{j}
$$

and the solution is

$$
w_{j}=\sum_{k=1}^{N-1} C_{A_{N-1} j, k}^{-1} \varepsilon_{j}
$$

and

$$
v_{\varepsilon}=\sum_{j, k} C_{A_{N-1} j, k}^{-1} \varepsilon_{k} J_{j}
$$

We have thus proved the following proposition.

Proposition 2: All the solutions of the equations of motion (2.2) determined by the ansätze (3.1), (3.2), and (2.3) are given by

$$
\begin{gathered}
h(r)=\operatorname{arv}_{\varepsilon}, \\
v_{\varepsilon}=\sum_{j, k} C_{A_{N-1}-1, k} \varepsilon_{k} J_{j},
\end{gathered}
$$

where $a$ is a real constant and $\varepsilon_{j}$ are signs, with $\varepsilon_{1}=1$.

These solutions are only local solutions, which means that they solve the differential equations. They do not extend automatically to global solutions, which are solutions with a well-defined baryon number. Looking for global solutions is the task of the next section.

\section{GLOBAL SOLUTIONS}

Up to now we have found the most general solution of the differential Skyrme equation. Nevertheless, it is not sufficient to determine a skyrmion, since global conditions have to be imposed in order to get a solution with a welldefined topological charge. This condition is not simply equivalent to imposing that the topological charge must be integer (this is just a consequence of the right topological condition) but that it has to wrap a homological cycle an entire number of times (mathematically, it has to cover a

\footnotetext{
${ }^{5} \mathrm{We}$ omit an irrelevant additive integration constant.
} 
cycle, which means to be a surjective map with a welldefined degree). We normalize the parametrizations so that all ranges are in $[0,2 \pi]$.

\section{A. Statement of the problem}

The difficulty in passing from local solutions to global solutions is twofold. In order to illustrate it, let us consider the specific example of $S U(4)$ when $k$ is given by $c_{i}=1$. For getting a well-defined global solution, the function

$$
g(\gamma)=e^{m \gamma k}
$$

is expected to provide a good coordinate of the image of the solution. Since the target space of the map is compact, this requires that if we extend the range of $\gamma$ to the whole $\mathbb{R}$, $g(\gamma)$ must result in a periodic function. Now, a simple calculation shows that the eigenvalues of $k$ are $\pm \mu_{+}, \pm \mu_{-}$, with

$$
\mu_{ \pm}=\frac{i}{2}(\sqrt{5} \pm 1) .
$$

This means that, for a suitable unitary constant matrix $U$, we have

$$
g(\gamma)=U \operatorname{diag}\left(e^{m \gamma \mu_{+}}, e^{-m \gamma \mu_{+}}, e^{m \gamma \mu_{-}}, e^{-m \gamma \mu_{-}}\right) U^{\dagger} .
$$

In particular, its elements have periodicities $T_{ \pm}$with

$$
T_{ \pm}=\frac{2 \pi}{m\left|\mu_{ \pm}\right|}
$$

But since

$$
\frac{T_{+}}{T_{-}}=\frac{1}{2}(3+\sqrt{5})
$$

is not rational, they do not have a common period and the orbit never closes; thus, it is not a periodic function but, rather, its orbit describes a curve which densely covers a bitorus in $S U(4)$. In particular, it is not possible to use $g(\gamma)$ as a good factor to get a finite covering of a cycle, even though it gives a solution of the equations of motion. It does not provide a solution with a well-defined topological number and must be discarded. One has to tackle the problem of looking for acceptable matrices $k$ that are matrices generating a well-defined period.

Assuming we have solved the periodicity problem, there is a second subtlety to be tackled: how to determine the right range of the coordinates in order to correctly cover a cycle. First, notice that $\pi_{3}(S U(N))=\mathbb{Z}$. This suggests that, homotopically, we have just one representative for any given topological (baryonic) charge. Moreover, since $\pi_{2}(S U(N))=0$, we also have $H_{3}(S U(N), \mathbb{Z})=\mathbb{Z}$, so we also have a unique homological representative. Nevertheless, the solutions do not have to be identified under deformation but, at most, under gauge equivalence. But since the action is not gauge invariant, in our case all the different representatives in a given equivalence class must be considered as different solutions.

We distinguish three different classes of solutions. The first two classes have canonical representatives: the $S U(2)$ type, which belong in every class, and the $S O(3)$ type, which belong in even classes only. They can be simply understood as follows. For any given $N$ we can embed the representations of $s u(2)$ into $s u(N)$. Exponentiating, they will give realizations of $S U(2)$ or $S O(3)$, depending on the specific representation. These realizations give rise to pure $S U$ (2)-type or $S O$ (3)-type solutions. However, they can be continuously deformed by varying the corresponding $\underline{c}$ when allowed, giving rise to solutions that are not embeddings; thus, we can consider them as true $S U(N)$ solutions. But there exists a third class of solutions that cannot be obtained as continuous deformations of embeddings. Their existence is due to the fact that $S U(N)$ has a center isomorphic to $\mathbb{Z}_{N}$, which acts continuously on $S U(N)$; see Appendix A. In particular, if $\Gamma$ is a normal subgroup of the center, then one can construct the group $S U(N)_{\Gamma}:=$ $S U(N) / \Gamma$. The new class of solutions is generated by cycles in $S U(N)$ that reduce to cycles of $S U(N)_{\Gamma}$ after the quotient. We call them genuine $S U(N)$ solutions. We consider them carefully in the explicit examples of $S U(3)$ and $S U(4)$, where everything is exactly computable, but now we briefly describe the $S U(2)$-type and $S O(3)$-type solutions, where some details are a priori known; see Appendix C.

An $S U(2)$-type cycle has the form

$$
U(\phi, \gamma, \theta)=e^{\phi k} e^{h^{\prime} r} e^{m \gamma k},
$$

where $h^{\prime}$ is constant and the coordinate must run as follows. The range of $r$ must be $T / 4$, where $T$ is the period of $e^{h^{\prime} r}$. The range of $\gamma$ must be $T_{k}$, the period of $e^{\gamma k}$ (with $m=1$ !), and the range of $\phi$ must be $T_{k} / 2$. Therefore, the convenient choice for the coordinates is

$$
\varphi \in\left[0, T_{k} / 2\right], \quad r \in[0, T / 4], \quad \gamma \in\left[0, T_{k}\right],
$$

corresponding to the baryon number

$$
B=m B_{0},
$$

where $B_{0}$ is the fundamental charge of the given skyrmion.

For $S O$ (3)-type cycles the interval for $\phi$ must cover an integer period, so the ranges must be

$$
\varphi \in\left[0, T_{k}\right], \quad r \in[0, T / 2], \quad \gamma \in\left[0, T_{k}\right],
$$

and the corresponding baryon number is

$$
B=2 m B_{0} .
$$


The $S O$ (3)-type can be defined as the "dibaryon class" after the seminal works [31,32]. These results were extended, keeping spherical symmetry, to the $S U(N)$ case in Refs. [53-56] leading to numerical nonembedded configurations in the $S U(N)$ Skyrme model. In the present paper we generalize those findings to the nonspherical case at finite baryon density, achieving, moreover, analytic solutions.

\section{B. $S U(3)$ skyrmions}

Let us apply the above formalism to the case $N=3$. In this case the problem of periodicity will not arise.

\section{1. $S O(3)$-type solutions and genuine $S U(3)$ solutions}

The matrix $k$ is

$$
k_{\underline{c}}=\left(\begin{array}{ccc}
0 & c_{1} & 0 \\
-c_{1}^{*} & 0 & c_{2} \\
0 & -c_{2}^{*} & 0
\end{array}\right) .
$$

We put $\|\underline{c}\|^{2}=\left|c_{1}\right|^{2}+\left|c_{2}\right|^{2}$. Then, the characteristic equation is

$$
\left(\lambda^{2}+\|\underline{c}\|^{2}\right) \lambda=0 .
$$

The eigenvalues are $\lambda_{0}=0$ and $\lambda_{ \pm}= \pm i\|\underline{\underline{c}}\|$, so that

$$
g(\gamma)=e^{\gamma k_{\underline{c}}}
$$

is periodic with period

$$
T_{k}=\frac{2 \pi}{\|\underline{c}\|} .
$$

Now, we determine the Cartan element. We have two possibilities according to the two possible choices for $\underline{\varepsilon}$ :

$$
\underline{\varepsilon}_{ \pm}=\left(\begin{array}{c}
1 \\
\pm 1
\end{array}\right) .
$$

The inverse Cartan matrix for $S U(3)$ is

$$
C_{A_{2}}^{-1}=\frac{1}{3}\left(\begin{array}{ll}
2 & 1 \\
1 & 2
\end{array}\right)
$$

Thus, we find the two solutions

$$
\begin{aligned}
& h_{+}(r)=\operatorname{ar}\left(J_{1}+J_{2}\right), \\
& h_{-}(r)=\frac{a}{3} r\left(J_{1}-J_{2}\right) .
\end{aligned}
$$

The period of $\exp h_{+}(r)$ is

$$
T_{h_{+}}=\frac{2 \pi}{a},
$$

while the one of $\exp h_{+}(r)$ is

$$
T_{h_{-}}=\frac{6 \pi}{a} .
$$

Now, we discuss the global properties in order to fix the ranges of the parameters. To this end, according to Appendix $\mathrm{C}$, we have to look for the intersection between the orbit of $h_{ \pm}$and the one of $\gamma k_{\underline{c}}$. Using the characteristic equation we immediately see that (see Appendix E 1)

$$
e^{\gamma k_{\underline{c}}}=I+\frac{\sin (\|\underline{c}\| \gamma)}{\|\underline{c}\|} k_{\underline{c}}+2 \frac{\sin ^{2}\left(\frac{\|\underline{\underline{c}}\|}{2} \gamma\right)}{\|\underline{c}\|^{2}} k_{\underline{c}}^{2},
$$

so that the intersection we are looking for is just the unit matrix $I$. However, we notice that the orbit of $\exp h_{-}(r)$ contains the elements

$\exp h_{-}(2 \pi / a)=e^{\frac{2}{3} \pi i} I, \quad \exp h_{-}(4 \pi / a)=e^{\frac{4}{3} \pi i} I$,

which are both in the center of $S U(3)$. Following Appendix $\mathrm{C}$, we conclude that $h_{-}(r)$ defines a genuine $S U$ (3) solution, while only $h_{+}(r)$ is of $S O(3)$-type.

In order to correctly define the solution, we thus have to identify the ranges as follows. First, it is convenient to normalize $\underline{c}$ so that $\|\underline{c}\|=1$. This is equivalent to rescaling the coordinates $\Phi$ and $\gamma$. Therefore, we fix once for all the metric to be

$$
d s^{2}=-d t^{2}+L_{r}^{2} d r^{2}+L_{\gamma}^{2} d \gamma^{2}+L_{\varphi}^{2} d \varphi^{2},
$$

with a range of coordinates

$$
0 \leq r \leq 2 \pi, \quad 0 \leq \gamma \leq 2 \pi, \quad 0 \leq \varphi \leq 2 \pi,
$$

with the caveat that, despite the chosen values, none of the coordinates is periodic. Our skyrmions are living in a rectangular box.

SO(3)-type solutions. - We already know that $r$ must cover $1 / 2$ of the period of the Cartan torus, which implies that we have to fix $a=\frac{1}{2}$. Hence, our solutions are

$$
\begin{gathered}
U_{ \pm}^{\underline{c}}[t, r, \varphi, \gamma]=e^{\Phi k_{c}} e^{\operatorname{ar}\left(J_{1} \pm J_{2}\right)} e^{m \gamma k_{c}}, \\
\Phi=\frac{t}{L_{\varphi}}-\varphi, \\
\varphi, \gamma, r \in[0,2 \pi], \\
B=2 m .
\end{gathered}
$$


More explicitly,

$$
U_{+}^{\underline{c}}[t, r, \varphi, \gamma]=\left(I+\sin (\Phi) k_{\underline{c}}+2 \sin ^{2} \frac{\Phi}{2} k_{\underline{c}}^{2}\right) \operatorname{diag}\left(e^{i \frac{r}{2}}, 1, e^{-i \frac{r}{2}}\right)\left(I+\sin (m \gamma) k_{\underline{c}}+2 \sin ^{2} \frac{m \gamma}{2} k_{\underline{c}}^{2}\right) .
$$

We can now compute the energy and the factor $g_{+}=\frac{E}{2 m}$. We omit details here, since particular cases of the general one for generic $N$ are considered below. We get

$$
g_{+}(m, \underline{c})=L_{r} L_{\gamma} L_{\varphi} \frac{K \pi^{3}}{m}\left[\frac{4}{L_{\phi}^{2}}+\frac{1}{8 L_{r}^{2}}+\frac{\lambda}{16 L_{\phi}^{2} L_{r}^{2}}+\frac{m^{2}}{L_{\gamma}^{2}}\left(2+\frac{\lambda}{32 L_{r}^{2}}+\frac{2 \lambda}{L_{\phi}^{2}}\left(1-3\left|c_{1}\right|^{2}\left|c_{2}\right|^{2}\right)\right)\right],
$$

where $\left|c_{1}\right|^{2}+\left|c_{2}\right|^{2}=1$. In particular, for each value of $m,\left|g_{+}(m, \underline{c})\right|$ takes its minimum at $\left|c_{1}\right|=\left|c_{2}\right|$, which is

$$
g_{+}(m, \underline{c})=L_{r} L_{\gamma} L_{\varphi} \frac{K \pi^{3}}{m}\left[\frac{4}{L_{\phi}^{2}}+\frac{1}{8 L_{r}^{2}}+\frac{\lambda}{16 L_{\phi}^{2} L_{r}^{2}}+\frac{m^{2}}{L_{\gamma}^{2}}\left(2+\frac{\lambda}{32 L_{r}^{2}}+\frac{\lambda}{2 L_{\phi}^{2}}\right)\right] .
$$

Some comments are in order here. The reason that the solution we have just described is of $S O(3)$-type can be understood by remembering that we are working with $3 \times 3$ matrices, which naturally carry a representation of spin 1 of the rotation group. Indeed, the minimum energy case just discussed, in which $\left|c_{j}\right|=1 / \sqrt{2}$, corresponds exactly to the case when the matrices $h_{+}$and $k_{\underline{c}}$ are the generators of the group $S O(3)$ in the representation of spin 1. The other solutions, for every fixed $m$, are continuous deformations obtained by varying $\underline{c}$, which does not change their topological nature, and, in particular, the baryon number, but it changes the energy. One can easily check that for generic $\underline{c}$ the matrices $h_{+}$and $k_{\underline{c}}$ do not generate a subgroup. One may wonder if this is related to the fact that their energy is not a minimum. The present remark suggests how to look for $S U(2)$-type solutions.

Genuine SU(3)-type solutions.-Since this case does not enter in the canonical classes, we have to manage separately the determination of the correct ranges (then normalized to $2 \pi$ as specified above). As for $r$, we will prove in Proposition 3 that in order to have $r$ in the range $[0,2 \pi]$, one has to fix $a=\frac{1}{2}$. For what concerns the other coordinates, let us note that $h_{-}(r)$ does not commute with $k_{\underline{c}}$ but it commutes with $k_{\underline{c}}^{2}$. Therefore, for $g(\gamma)=e^{\gamma k_{\underline{c}}}$, we see that $g\left(T_{k} / 2\right)$ commutes with $e^{h_{-}(r)}$. This means that we can write

$$
g\left(\Phi+T_{k} / 2\right) e^{h_{-}(r)} g(\gamma)=g(\Phi) g\left(T_{k} / 2\right) e^{h_{-}(r)} g(\gamma)=g(\Phi) e^{h_{-}(r)} g\left(T_{k} / 2\right) g(\gamma)=g(\Phi) e^{h_{-}(r)} g\left(\gamma+T_{k} / 2\right) .
$$

If we assume that $U^{\underline{c}}[\Phi, r, \gamma]=g(\Phi) e^{h_{-}(r)} g(\gamma)$ is covering a cycle, the relation $U^{\underline{c}}\left[\Phi+T_{k} / 2, r, \gamma\right]=U^{\underline{c}}\left[\Phi, r, \gamma+T_{k} / 2\right]$ shows that we are covering it twice unless we restrict one of the two ranges, of $\Phi$ and of $\gamma$, to one-half the period of $g$. We choose to reduce $\Phi$, so we replace $\Phi$ with $\Phi / 2$. Thus, our solution is

$$
\begin{gathered}
U^{\underline{c}}[t, r, \varphi, \gamma]=e^{\frac{\Phi}{2} k_{c}} e^{\operatorname{ar}\left(J_{1} \pm J_{2}\right)} e^{m \gamma k_{\underline{c}}}, \\
\Phi=\frac{t}{L_{\varphi}}-\varphi \\
\varphi, \gamma, r \in[0,2 \pi], \\
B=m
\end{gathered}
$$

where $B$ has been computed as in Appendix F. Explicitly,

$$
U^{\underline{\underline{c}}}[t, r, \varphi, \gamma]=\left(I+\sin \frac{\Phi}{2} k_{\underline{c}}+2 \sin ^{2} \frac{\Phi}{4} k_{\underline{c}}^{2}\right) \operatorname{diag}\left(e^{i \frac{r}{\sigma}}, e^{-i \frac{r}{3}}, e^{i \frac{r}{6}}\right)\left(I+\sin (m \gamma) k_{\underline{c}}+2 \sin ^{2} \frac{m \gamma}{2} k_{\underline{c}}^{2}\right) .
$$

For $U_{-}, g$ is independent from $\underline{c}$,

$$
g_{-}(m, \underline{c})=L_{r} L_{\gamma} L_{\varphi} \frac{K \pi^{3}}{2 m}\left[\frac{4}{L_{\phi}^{2}}+\frac{2}{3 L_{r}^{2}}+\frac{\lambda}{4 L_{\phi}^{2} L_{r}^{2}}+8 \frac{m^{2}}{L_{\gamma}^{2}}\left(1+\frac{\lambda}{16 L_{r}^{2}}+\frac{\lambda}{4 L_{\phi}^{2}}\right)\right] .
$$




\section{2. $S U(2)$-type solutions}

It is now clear that in order to find $S U(2)$-type solutions we have to consider deformations of spin $\frac{1}{2}$ representations. This can be obtained by "reducing matrices" down to $2 \times 2$, and it can be achieved by choosing

$$
k \equiv k_{c}=\left(\begin{array}{ccc}
0 & c & 0 \\
-c^{*} & 0 & 0 \\
0 & 0 & 0
\end{array}\right)
$$

where $c$ is a phase. This is not the same thing as simply putting $c_{2}=0$ in $k_{\underline{c}}$ in the sense that we have to choose $k=k_{c}$ before solving Eq. (3.9). Indeed, in Eq. (3.9) we assumed that all simple roots are involved. This fixes the set of possible choices of $h(r)$, and if in the above solutions we deform smoothly $\underline{c}$ to $(c, 0)$, we cannot move away from our topological classes. This is confirmed by the fact that if we put $c_{2}=0$, the matrix $k$ reduces to a $2 \times 2$ matrix, but the $k_{ \pm}$do not allow us to reduce the representation down to $\mathbb{C}^{2}$. We have to make a discontinuous deformation. The point is that for $c_{2}=0$ the root $\alpha_{2}$ does not enter into Eq. (3.9), which, indeed, for $N=3$ becomes just an identity. This means that when $c_{2}=0$ we can choose for $h(r)$ any combination

$$
h(r)=a r J_{1}+b r J_{2},
$$

with the only caveat that $e^{h(r)}$ must be periodic, so $a$ and $b$ must be in a rational ratio. We can set

$$
h_{q}(r)=\operatorname{arJ}_{1}+a q r J_{2}, \quad q \in \mathbb{Q} .
$$

For $q= \pm 1$ we return to the previous $S O(3)$ solutions, while, of course, $q=0$ provides a canonical embedding of $S U$ (2) into $S U$ (3), thus identifying an $S U$ (2)-type solution. It is worthwhile to mention that since $q \in \mathbb{Q}$, it cannot be deformed continuously among the three values, which is compatible with the fact that the case $q=0$ is not in the same topological class as the other ones and, indeed, we may wonder what happens for all the other values of $q$ since they would generate new genuine $S U(3)$ solutions. However, they have vanishing baryon number, so we will not consider them further.

Thus, we get the solutions

$$
\begin{gathered}
U_{0}^{c}[t, r, \varphi, \gamma]=e^{\frac{1}{2} \Phi k_{c}} e^{\frac{r}{4} J_{1}} e^{n \gamma k_{c}}, \\
\Phi=\frac{t}{L_{\varphi}}-\varphi, \\
\varphi, \gamma, r \in[0,2 \pi], \\
B=n .
\end{gathered}
$$

The $1 / 2$ factor in the first exponent has been added to ensure that when $\Phi$ varies in $[0,2 \pi]$, it covers half of the period. Finally, we can compute the factor $g$ :

$$
g_{0}(n, c)=\frac{K \pi^{3}}{n}\left[\frac{2}{L_{\phi}^{2}}+\frac{1}{4 L_{r}^{2}}+\frac{\lambda}{8 L_{\phi}^{2} L_{r}^{2}}+\frac{n^{2}}{L_{\gamma}^{2}}\left(4+\frac{\lambda}{4 L_{r}^{2}}+\frac{\lambda}{L_{\phi}^{2}}\right)\right] .
$$

\section{C. $S U(N)$ skyrmions}

We now consider the class of skyrmions associated with the matrix $k$ given by

$$
k_{\underline{c}}=\sum_{j=1}^{N-1}\left(c_{j} E_{j, j+1}-c_{j}^{*} E_{j+1, j}\right) .
$$

We will limit ourselves to the case when all the $c_{j}$ are different from zero. Here, we have to face the problem of establishing for which choices of $c_{j}$ the matrix $e^{\gamma k_{c}}$ is periodic. For now, let us assume this problem is solved and write down the corresponding solution:

$$
\begin{aligned}
& U_{\bar{\varepsilon}}^{\underline{c}}[t, r, \varphi, \gamma]=e^{\sigma \Phi k_{\underline{c}}} e^{a v_{\varepsilon} r} e^{m \gamma k_{\underline{c}}}, \\
& \Phi=\frac{t}{L_{\varphi}}-\varphi,
\end{aligned}
$$

$$
\begin{gathered}
\varphi, \gamma, r \in[0,2 \pi], \\
B=\sigma 2 m\|\underline{c}\|^{2},
\end{gathered}
$$

where $\sigma=1$ for $S O(3)$-type solutions and $\sigma=1 / 2$ for $S U$ (2)-type solutions, and $v_{\varepsilon}$ is given by Eq. (3.21). For general genuine solutions the value of $\sigma$ must be computed case by case. For any admissible $\underline{c}$ these are $2^{N-2}$ solutions (since $\varepsilon_{1}=1$ ). In principle, $a$ could depend on $N$ and $\underline{\varepsilon}$. However, we now show that this is not the case and that the value of $a$ is completely fixed by requiring that the normalized interval $[0,2 \pi]$ for $r$ must have the extension necessary to cover a cycle once:

Proposition 3: If $\exp \left(a v_{\varepsilon} r\right)$ is such that $r \in[0,2 \pi]$, and the corresponding map $\bar{U} \frac{c}{\bar{\varepsilon}}[t, r, \varphi, \gamma]$ does not have to cover a cycle more than once, then necessarily $a=\frac{1}{2}$.

Proof.-The proof is simply based on the same strategy used in Ref. [39]: One first constructs the invariant 
measure restricted to the hypothetical cycle; the resulting measure will depend explicitly on some of the coordinates and will vanish at a specific value of that coordinate. The good range for such a coordinate to cover a cycle just once is any range between two vanishing points. The Haar measure that is restricted to a cycle, apart from an eventual normalization constant, is just $\rho_{B}$, which is computed in Appendix F. Since it depends on $r$ via $\sin (a r)$, we see that a suitable interval for $r$ is $[0, \pi / a]$. Since we want it to be $[0,2 \pi]$, it must be $a=\frac{1}{2}$.

Therefore, we definitely have

$$
a=\frac{1}{2}
$$

in any case. Now, we can compute the $g$-factor for our solutions. To this end, first note that

$$
\begin{aligned}
T_{00} & =-\frac{K}{2} \operatorname{Tr}\left(\frac{1}{2}\left(R^{\gamma} R_{\gamma}+R^{r} R_{r}\right)+\frac{\lambda}{16} F_{\rho \sigma} F^{\rho \sigma}+R_{t} R_{t}+\frac{\lambda}{4} g^{\alpha \beta} F_{t \alpha} F_{t \beta}\right) \\
& =-\frac{K}{4} \operatorname{Tr}\left(\frac{R_{\gamma}^{2}}{L_{\gamma}^{2}}+\frac{R_{r}^{2}}{L_{r}^{2}}\right)-\frac{K \lambda}{16} \operatorname{Tr}\left(F_{\gamma r}\right)^{2}-\frac{K}{2} \operatorname{Tr} R_{t}^{2}-\frac{K \lambda}{8 L_{\varphi}^{2}} \operatorname{Tr}\left(\frac{F_{\Phi r}^{2}}{L_{r}^{2}}+\frac{F_{\Phi \gamma}^{2}}{L_{\gamma}^{2}}\right) .
\end{aligned}
$$

according to Appendix B, and we use

$$
R_{t}=\frac{1}{L_{\varphi}} R_{\Phi}, \quad F_{t \alpha}=\frac{1}{L_{\varphi}} F_{\Phi \alpha} .
$$

According to Eqs. (B6)-(B8), with $a=\frac{1}{2}$, we have

$$
\begin{gathered}
\operatorname{Tr} R_{t}^{2}=\frac{\sigma^{2}}{L_{\varphi}^{2}} \operatorname{Tr} k_{\underline{c}}^{2}=-\frac{2}{L_{\varphi}^{2}}\|\underline{c}\|^{2} \sigma^{2}, \\
\operatorname{Tr} R_{\gamma}^{2}=m^{2} \operatorname{Tr} k_{\underline{c}}^{2}=-2 m^{2}\|\underline{c}\|^{2}, \\
\operatorname{Tr} R_{r}^{2}=\frac{1}{4} \operatorname{Tr} v_{\underline{\varepsilon}}^{2}=-\frac{1}{4} \sum_{j, k} C_{A_{N-1}-1} \varepsilon_{j} \varepsilon_{k} \equiv-\frac{1}{4}\left\|v_{\underline{\varepsilon}}\right\|^{2}, \\
\operatorname{Tr}\left(F_{\gamma r}\right)^{2}=m^{2} \operatorname{Tr}\left(\left[h^{\prime}, k_{\underline{c}}\right]\right)^{2}=-\frac{m^{2}}{2}\|\underline{c}\|^{2}, \\
\operatorname{Tr}\left(F_{\phi r}\right)^{2}=\sigma^{2} \operatorname{Tr}\left(\left[x, h^{\prime}\right]^{2}\right)=-\frac{\sigma^{2}}{2}\|\underline{c}\|^{2}, \\
\operatorname{Tr}\left(F_{\phi \gamma}\right)^{2}=\sigma^{2} m^{2} \operatorname{Tr}\left(\left[x, k_{\underline{c}}\right]\right)^{2}=-8 m^{2} \sigma^{2} \sin ^{2} \frac{r}{2}\left(\sum_{j=1}^{N-1}\left|c_{j}\right|^{4}+\sum_{j=1}^{N-2}\left|c_{j}\right|^{2}\left|c_{j+1}\right|^{2} \frac{1}{2}\left(1-3 \varepsilon_{j} \varepsilon_{j+1}\right)\right) .
\end{gathered}
$$

Replacing this in the expression for $T_{00}$ and using that the energy is

$$
E=\int_{0}^{2 \pi} d r \int_{0}^{2 \pi} d \varphi \int_{0}^{2 \pi} d \gamma L_{r} L_{\varphi} L_{\gamma} T_{00}(r),
$$

we get

$$
\begin{aligned}
E= & L_{r} L_{\gamma} L_{\phi}\|\underline{c}\|^{2} \frac{K}{2} \pi^{3}\left[16 \frac{\sigma^{2}}{L_{\varphi}^{2}}+\frac{\left\|v_{\underline{\varepsilon}}\right\|^{2}}{\|\underline{c}\|^{2} L_{r}^{2}}+\frac{\sigma^{2} \lambda}{L_{\varphi}^{2} L_{r}^{2}}\right. \\
& \left.+8 \frac{m^{2}}{L_{\gamma}^{2}}\left(1+\frac{\lambda}{16 L_{r}^{2}}+\frac{\lambda \sigma^{2}}{L_{\varphi}^{2}\|\underline{c}\|^{2}}\left(\sum_{j=1}^{N-1}\left|c_{j}\right|^{4}+\sum_{j=1}^{N-2}\left|c_{j}\right|^{2}\left|c_{j+1}\right|^{2}\left(\frac{1}{2}-\frac{3}{2} \varepsilon_{j} \varepsilon_{j+1}\right)\right)\right)\right] .
\end{aligned}
$$

In a similar way, one can compute the baryon number. This is done in Appendix F, with the result 


$$
B=2 m \sigma\|\underline{c}\|^{2} .
$$

From these results we immediately get the $g$-factor:

$$
\begin{aligned}
g(N, m, \underline{c}, \varepsilon)= & L_{r} L_{\gamma} L_{\phi} \frac{K \pi^{3}}{4 \sigma m}\left[16 \frac{\sigma^{2}}{L_{\varphi}^{2}}+\frac{\left\|v_{\underline{\varepsilon}}\right\|^{2}}{\|\underline{c}\|^{2} L_{r}^{2}}+\frac{\sigma^{2} \lambda}{L_{\varphi}^{2} L_{r}^{2}}\right. \\
& \left.+8 \frac{m^{2}}{L_{\gamma}^{2}}\left(1+\frac{\lambda}{16 L_{r}^{2}}+\frac{\lambda \sigma^{2}}{L_{\varphi}^{2}\|\underline{c}\|^{2}}\left(\sum_{j=1}^{N-1}\left|c_{j}\right|^{4}+\sum_{j=1}^{N-2}\left|c_{j}\right|^{2}\left|c_{j+1}\right|^{2}\left(\frac{1}{2}-\frac{3}{2} \varepsilon_{j} \varepsilon_{j+1}\right)\right)\right)\right] .
\end{aligned}
$$

Up to now, we have assumed $\underline{\underline{c}}$ to be normalized so that $g(\gamma)=e^{\gamma k_{\underline{c}}}$ has period $2 \pi$. However, we will not find a solution until we are able to specify for which $\underline{c}$ the function $g$ is periodic. Therefore, we cannot further postpone tackling this problem.

However, before considering it, in general, we concentrate on a very particular case, when $\varepsilon_{j}=1$ for all $j$. In this case

$$
\begin{aligned}
g(N, m, \underline{c})= & L_{r} L_{\gamma} L_{\phi} \frac{K \pi^{3}}{4 \sigma m}\left[16 \frac{\sigma^{2}}{L_{\varphi}^{2}}+\frac{\|v\|^{2}}{\|\underline{c}\|^{2} L_{r}^{2}}+\frac{\sigma^{2} \lambda}{L_{\varphi}^{2} L_{r}^{2}}\right. \\
& \left.+8 \frac{m^{2}}{L_{\gamma}^{2}}\left(1+\frac{\lambda}{16 L_{r}^{2}}+\frac{\lambda \sigma^{2}}{L_{\varphi}^{2}\|\underline{c}\|^{2}}\left(\sum_{j=1}^{N-1}\left|c_{j}\right|^{4}-\sum_{j=1}^{N-2}\left|c_{j}\right|^{2}\left|c_{j+1}\right|^{2}\right)\right)\right] .
\end{aligned}
$$

It is clear that, among all possible choices for $\varepsilon_{j}$, this minimizes the energy, apart from possible effects due to $\|v\|$. We also want minimize with respect to the $c_{j}$, assuming the normalization of $\|\underline{c}\|$ is fixed. Introducing a Lagrange multiplier $\Lambda$, we have to extremize the function

$$
f(\underline{c})=\sum_{j=1}^{N-1}\left|c_{j}\right|^{4}-\sum_{j=1}^{N-2}\left|c_{j}\right|^{2}\left|c_{j+1}\right|^{2}-\Lambda\|\underline{c}\|^{2} .
$$

Deriving with respect to $\left|c_{j}\right|^{2}$, we get the system

$$
C_{A_{N-1}} \underline{|c|^{2}}=\Lambda \underline{1}
$$

with 1 being the vector in $\mathbb{R}^{N-1}$ having all elements equal to 1. This gives the solution

$$
\left|c_{j}\right|^{2}=\frac{\Lambda}{2} j(N-j)
$$

Interestingly, this also automatically solves the periodicity problem. It is easy to see (Appendix D) that

$c_{j}=\zeta_{j} \sqrt{\frac{\Lambda}{2} j(N-j)}, \quad \Lambda= \begin{cases}\frac{1}{2} & \text { for odd } N \\ 2 & \text { for even } N\end{cases}$

where $\zeta_{j}$ are arbitrary phases, given a matrix $e^{\gamma k_{\underline{c}}}$ that is periodic in $\gamma$ with period $2 \pi$. For $v$ we find

$$
v=\sum_{j, k} C_{A_{N-1 j, k}}^{-1} J_{j}
$$

Moreover, we have the following proposition.

Proposition 4: If $c_{j}$ are given by Eq. (4.58) and $v$ is as in Eq. (4.59), then

$$
\begin{aligned}
& \|\underline{c}\|^{2}=\frac{\Lambda}{12} N\left(N^{2}-1\right), \\
& \|v\|^{2}=\frac{1}{12} N\left(N^{2}-1\right),
\end{aligned}
$$

and

$$
\begin{aligned}
\sum_{j=1}^{N-1}\left|c_{j}\right|^{4}-\sum_{j=1}^{N-2}\left|c_{j}\right|^{2}\left|c_{j+1}\right|^{2} & =\frac{\Lambda^{2}}{24} N\left(N^{2}-1\right) \\
& =\frac{\Lambda}{2}\|\underline{c}\|^{2} .
\end{aligned}
$$

Proof.-The first result follows immediately from the well-known formulas

$$
\begin{gathered}
\sum_{j=1}^{N-1} j=\frac{N(N-1)}{2}, \\
\sum_{j=1}^{N-1} j^{2}=\frac{N(N-1)(2 N-1)}{6} .
\end{gathered}
$$

For the second expression notice that, by using Eq. (A15), 


$$
\begin{aligned}
\|v\|^{2} & =\sum_{j, k} C_{A_{N-1} j, k}^{-1} \\
& =\frac{1}{N}\left[\sum_{j<k} j(N-k)+\sum_{j \geq k} k(N-j)\right] \\
& =-\frac{1}{N} \sum_{j, k} j k+\sum_{j<k} j+\sum_{j \geq k} k \\
& =-\frac{1}{N}\left(\sum_{j=1}^{N-1} j\right)^{2}+\sum_{j=1}^{N-1} j(N-j-1)+\sum_{k=1}^{N-1} k(N-k),
\end{aligned}
$$

and the final expression again follows after applying the above well-known formulas.

For the last formula, notice that the $c_{j}$ are solutions of

$$
\frac{\partial f}{\partial c_{k}}=0, \quad k=1, \ldots, N-1
$$

where $f$ is given by Eq. (4.55). From this we get

$$
\sum_{k=1}^{N-1} c_{k} \frac{\partial f}{\partial c_{k}}=0
$$

Now, $f$ is the sum of two homogeneous pieces, one of degree 4 and the other of degree 2 . Therefore, we can use the Euler theorem ${ }^{6}$ to rewrite the last as

$$
0=4\left(\sum_{j=1}^{N-1}\left|c_{j}\right|^{4}-\sum_{j=1}^{N-2}\left|c_{j}\right|^{2}\left|c_{j+1}\right|^{2}\right)-2 \Lambda\|\underline{c}\|^{2},
$$

which completes the proof.

Using these results and noticing that $\sigma^{2} \Lambda=1 / 2$, we find for the energy per baryon

$$
\begin{gathered}
g(N, m)_{\min }=L_{r} L_{\gamma} L_{\varphi} \frac{K \pi^{3}}{\sigma m}\left[4 \frac{\sigma^{2}}{L_{\varphi}^{2}}+\frac{\sigma^{2}}{2 L_{r}^{2}}+\frac{\sigma^{2} \lambda}{4 L_{\varphi}^{2} L_{r}^{2}}+2 \frac{m^{2}}{L_{\gamma}^{2}}\left(1+\frac{\lambda}{16 L_{r}^{2}}+\frac{\lambda}{4 L_{\varphi}^{2}}\right)\right], \\
\Lambda=2^{(-1)^{N}}, \quad \sigma=2^{-\frac{(-1)^{N}+1}{2}}, \\
B=2^{\frac{1+(-1)^{N}}{2}} m \frac{1}{12} N\left(N^{2}-1\right) .
\end{gathered}
$$

Notice that $g(N, m)$ depends on $N$ only through $\sigma$.

We can also notice that

$$
I_{N}=\frac{N\left(N^{2}-1\right)}{6}
$$

is the Dynkin index of the given representation of the principal representation of $s l(2)$ in $\operatorname{sl}(N)$, so the fundamental baryonic charge associated with it is

$$
B=\frac{1}{2 \sigma} I_{N}
$$

Notice that for $N$ odd, $I_{N}$ is even; thus, $B$ is always integer.

Finally, we are also interested in minimizing expression (4.69) with respect to $L_{a}, a=\varphi, r, \gamma$. This is done, in general, in Appendix G. By using the formulas therein and the ones in the last proposition, we find that the minimum is reached at

\footnotetext{
${ }^{6}$ That is for a homogeneous function $f: \mathbb{R}^{N} \rightarrow \mathbb{R}$ of degree $L$, one has

$$
\vec{x} \cdot \operatorname{grad} f=L f .
$$
}

$$
L_{\varphi}=\frac{\sqrt{\lambda}}{2^{\frac{3}{4}}}, \quad L_{r}=\frac{\sqrt{\lambda}}{4}, \quad L_{\gamma}=\frac{m}{\sigma} \frac{\sqrt{\lambda}}{2^{\frac{5}{4}}},
$$

with the corresponding minimal value

$$
g_{\min }=K \sqrt{\lambda} \pi^{3}(1+2 \sqrt{2}) .
$$

Using normalized units [corresponding to $\lambda=1$ and $K=\left(6 \pi^{2}\right)^{-1}$ ] we get

$$
g_{\text {min,stand }}=\pi \frac{1+2 \sqrt{2}}{6} \approx 2.00456 .
$$

Notice that this is independent from $N$, and it is expected to be the absolute minimum with respect to any choice of $\varepsilon_{j}$. We will not try to prove this conjecture here; we will limit ourselves to checking it for $N=4$ below. The comparison with Ref. [57] is very interesting. The present results are slightly above the bound in Ref. [57] due to the time dependence in the ansatz. Note, however, that the present time dependence cannot be undone as the present solutions also wrap, in a topologically nontrivial way, around the time direction. To the best of our knowledge, this is the first analytic computation showing explicitly how the closeness 
to the BPS bound "evolves" with $N$ in the $S U(N)$ Skyrme model.

To be more specific, as has already been emphasized, we are interested in topologically nontrivial solutions. In the present context this means that we only consider $S U(N)$ ansatz such that

$$
\rho_{B}=\operatorname{Tr}\left(U^{-1} d U\right)^{3} \neq 0 .
$$

As has been discussed in the previous sections, $\rho_{B}$ represents the baryon density when it is nonvanishing along three-dimensional spacelike hypersurfaces $\Sigma_{t=\text { const }}$.

In these cases, the integral of $\rho_{B}$ over $\Sigma_{t=\text { const }}$ represents the baryon charge, while mathematically these integrals represent how many times the $S U(N)$-valued skyrmions wrap around $\Sigma_{t=\text { const. }}$ On the other hand, $\rho_{B}$ can be topologically trivial also along timelike hypersurfaces. In this case, one can also consider the wrapping of the $S U(N)$ valued configurations along three-dimensional timelike hypersurfaces. The configurations which have been constructed here are-as a direct check easily revealstopologically nontrivial in two ways. Not only do they possess nonvanishing baryonic charge, but they are also wrapped nontrivially along timelike hypersurfaces. Indeed, if one considers

$$
\begin{aligned}
U_{\bar{\varepsilon}}^{c}[t, r, \varphi, \gamma] & =e^{\sigma \Phi k_{\underline{c}}} e^{a v_{\varepsilon} r} e^{m \gamma k_{\underline{c}}}, \\
\Phi & =\frac{t}{L_{\varphi}}-\varphi
\end{aligned}
$$

then the corresponding topological density has one spacelike component and one timelike component:

$$
\rho_{B} \sim d r \wedge d \varphi \wedge d \gamma-d r \wedge d\left(\frac{t}{L}\right) \wedge d \gamma
$$

In particular, this implies that these $S U(N)$ skyrmions wrap nontrivially around the three-dimensional timelike $\{\varphi=$ const $\}$ hypersurfaces. The consequence of this fact is that the time dependence of the present configuration "cannot be undone"; otherwise the winding number corresponding to the $\{\varphi=$ const $\}$ hypersurfaces would change.

\section{Solving the periodicity problem}

The solution to this problem is provided in Appendix E. ${ }^{7}$ We discuss the main results here. The vectors $\underline{c} \in \mathbb{C}^{N-1}$, having all components different from zero and allowing for a periodic function $g(\gamma)=e^{\gamma k_{c}}$, with period $2 \pi$, form a family

$$
\underline{c}=\underline{c}(\underline{m}, \underline{\alpha}, \underline{t}),
$$

${ }^{7}$ S.L.C. is particularly grateful to Laurent Lafforgue for suggesting how to tackle this problem in full generality. where $\underline{m}=\left(m_{1}, \ldots, m_{n}\right)$, is a finite, strictly increasing sequence of strictly positive coprime integer numbers, $n$ is the integer part of $N / 2, \underline{\alpha} \in[0,2 \pi)^{N-1}$, and $\underline{t} \in W \subset$ $\mathbb{R}^{N-n-1}$ is a set of parameters parametrizing the strictly positive real solutions of the algebraic system

$$
\begin{gathered}
\sum_{j=1}^{N-1} \zeta_{j}=\sum_{a=1}^{n} m_{a}^{2}, \\
\sum_{j_{1} \ll \ldots \ll j_{k} \leq N-1} \zeta_{j_{1}} \cdots \zeta_{j_{k}}=\sum_{a_{1}<\ldots<a_{k} \leq n} m_{a_{1}}^{2} \cdots m_{a_{k}}^{2}, \\
k=2, \ldots, n,
\end{gathered}
$$

in real variables $\zeta_{j}, j=1, \ldots, N-1$.

The parameters $\underline{\alpha}$ and $\underline{t}$ form a moduli space $\mathbb{T}^{N-1} \times W$. The relevant physical quantities depend only on $\left|c_{j}\right|$, so they are independent on the components in the $N-1$ dimensional torus. Therefore, we can say that only $W$ represents the relevant moduli. As one could expect, in particular, the baryon number associated with a solution constructed with $\underline{c}(\underline{m}, \underline{\alpha}, \underline{t})$ depends only on $\underline{m}$ and not on the continuous moduli:

$$
B=2 \sigma m \sum_{a=1}^{n} m_{a}^{2}
$$

The general form of $g(\gamma)$ is

$$
e^{\gamma k_{\underline{c}}}=f_{0}(\gamma, \underline{m}) \mathbb{I}+\sum_{j=1}^{N-1} f_{j}(\gamma, \underline{m}) k_{\underline{c}(\underline{m}, \underline{\alpha}, \underline{t})}^{j},
$$

where the $f_{\beta}, \beta=0, \ldots, N-1$ are linear combinations of 1 and $\sin \left(m_{a} \gamma\right), \cos \left(m_{a} \gamma\right)$, with rational functions of $\underline{m}$ as coefficients, and satisfying $f_{0}(0, \underline{m})=1, f_{j}(0, \underline{m})=0$ for $j>0$. In particular, the dependence on the continuous moduli is only through $k_{\underline{c}}^{j}$.

\section{E. Back to $N=4$}

Following Appendix E3, for any two coprime positive integers $p$ and $q$ such that $p>q$, for $N=4$, we can find four families of solutions, each one parametrized by three real phases $\alpha_{1}, \alpha_{2}, \alpha_{3}$ and a real modulus $\tau \in[q, p]$. Each of these families is specified by one of the four possible inequivalent choices for the discrete vector $\varepsilon$. Recall that in this case the inverse Cartan matrix is

$$
C_{A_{3}}^{-1}=\frac{1}{4}\left(\begin{array}{lll}
3 & 2 & 1 \\
2 & 4 & 2 \\
1 & 2 & 3
\end{array}\right) .
$$

We also have (see Appendix E 3) 


$$
\begin{aligned}
g_{4}(x) \equiv e^{x k_{\underline{c}}}= & \left(\frac{p^{2}}{p^{2}-q^{2}} \cos (q x)-\frac{q^{2}}{p^{2}-q^{2}} \cos (p x)\right) \mathbb{I}+\left(\frac{p^{2}}{q\left(p^{2}-q^{2}\right)} \sin (q x)-\frac{q^{2}}{p\left(p^{2}-q^{2}\right)} \sin (p x)\right) k_{\underline{c}} \\
& +\left(\frac{1}{p^{2}-q^{2}}(\cos (q x)-\cos (p x))\right) k_{\underline{c}}^{2}+\left(\frac{1}{p^{2}-q^{2}}\left(\frac{\sin (q x)}{q}-\frac{\sin (p x)}{p}\right)\right) k_{\underline{c}}^{3},
\end{aligned}
$$

with

$$
\begin{aligned}
& k_{\underline{c}}=\left(\begin{array}{cccc}
0 & e^{i \alpha_{1}} \tau & 0 & 0 \\
-e^{-i \alpha_{1}} \tau & 0 & e^{i \alpha_{2}} \psi & 0 \\
0 & -e^{-i \alpha_{2}} \psi & 0 & \frac{p q}{\tau} e^{i \alpha_{3}} \\
0 & 0 & -\frac{p q}{\tau} e^{-i \alpha_{3}} & 0
\end{array}\right) \\
& k_{\underline{c}}^{2}=\left(\begin{array}{cccc}
-\tau^{2} & 0 & e^{i\left(\alpha_{1}+\alpha_{2}\right)} \tau \psi & 0 \\
0 & -\left(p^{2}+q^{2}-\frac{p^{2} q^{2}}{\tau^{2}}\right) & 0 & e^{i\left(\alpha_{2}+\alpha_{3}\right)} \frac{p q}{\tau} \psi \\
e^{-i\left(\alpha_{1}+\alpha_{2}\right)} \tau \psi & 0 & -\left(p^{2}+q^{2}-\tau^{2}\right) & 0 \\
0 & e^{-i\left(\alpha_{2}+\alpha_{3}\right) \frac{p q}{\tau} \psi} & 0 & -\frac{p^{2} q^{2}}{\tau^{2}}
\end{array}\right) \text {, }
\end{aligned}
$$

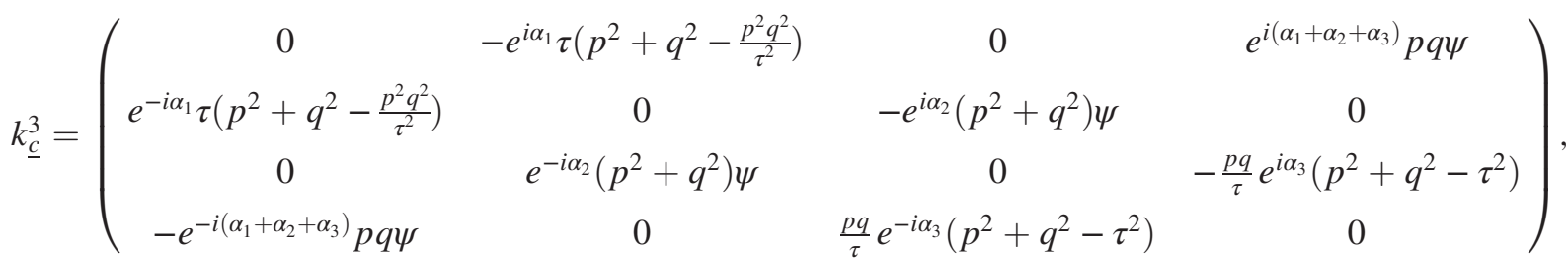

and

$$
\psi=\sqrt{p^{2}+q^{2}-\tau^{2}-\frac{p^{2} q^{2}}{\tau^{2}}} .
$$

\section{The almost $S U(2)$-type solutions}

The $S U(2)$ solution is expected to be identified by $\varepsilon_{a}=(1,1,1)$. Indeed, from Eq. (3.21) we have

$$
v_{a}=\frac{i}{2} \operatorname{diag}(3,1,-1,-3), \quad\left\|v_{a}\right\|^{2}=5,
$$

which is exactly the matrix representing the diagonal generator of $S U(2)$ in the spin-3/2 representation. However, this is not true, in general, and we see that in this series only the one with $(p, q)=(3,1)$ is deformable to an $S U(2)$ embedding. Let us first look at the coordinate ranges. Regarding the range of $r$, it is completely fixed by Proposition 3. As for the remaining ranges, they must correspond to the period of $g_{4}$, unless there are (finite discrete) subgroups of the $U(1)$ group generated by $g_{4}$, which commute with $v_{a}$. Since $v_{a}$ does not commute with $k_{\underline{c}}^{j}, j=1,2,3$ (or any linear combination thereof), we have to look for the values of $x$, such that $f_{j}(x)=0, j=1,2,3$ (Appendix E 3). Looking at $f_{3}$, this means

$$
\cos (p x)=\cos (q x)
$$

that is,

$$
p x= \pm q x+2 \ell \pi
$$

for some integer $\ell$. For the $x$ satisfying this condition, call them $x_{p} m$, one has for $f_{3}$

$$
f_{3}(x \pm)=\frac{1}{p^{2}-q^{2}}\left(\frac{1}{q}-\frac{1}{p}\right) \sin \left(q x_{ \pm}\right),
$$

which is zero for $x=j \pi$ for some integer $j$. Since our coordinates are forced to vary in $[0,2 \pi]$, the only nontrivial 
possibility is $x=\pi$. Putting this back into the previous condition, we must also have

$$
p-q=2 \ell,
$$

which means that, since $p$ and $q$ are coprime, this happens only when both $p$ and $q$ are odd. In this case $g_{4}(\pi)=-\mathbb{I}$. Therefore, we see that for $p-q$ odd, there are no discrete symmetries, and the ranges of $\Phi$ and $\gamma$ must coincide with the whole period, so $\sigma=1$. Instead, for $p-q$ even we have

$$
\begin{aligned}
& g_{4}(\Phi+\pi) e^{\frac{1}{2} v_{a} r} g_{4}(\gamma+\pi) \\
& =g_{4}(\Phi)(-\mathbb{I}) e^{\frac{1}{2} v_{a} r}(-\mathbb{I}) g_{4}(\gamma) \\
& =g_{4}(\Phi) e^{\frac{1}{2} v_{a} r} g_{4}(\gamma),
\end{aligned}
$$

so we see that, to any point on the image, there correspond two different coordinates $(\Phi, \gamma)$ and $(\Phi+\pi, \gamma+\pi)$, unless we restrict one of the two ranges to half a period. We choose to use $\Phi$, and, in order to keep its range as $[0,2 \pi]$, we fix $\sigma=1 / 2$.

The field is

$$
\begin{gathered}
U_{a}=g_{4}\left(\sigma_{p-q} \Phi\right)\left(\begin{array}{cccc}
e^{\frac{3}{4} i r} & 0 & 0 & 0 \\
0 & e^{\frac{1}{4} i r} & 0 & 0 \\
0 & 0 & e^{-\frac{1}{4} i r} & 0 \\
0 & 0 & 0 & e^{-\frac{3}{4} i r}
\end{array}\right) g_{4}(m \gamma) \\
\Phi=\frac{t}{L_{\varphi}}-\varphi \\
\sigma_{p-q}= \begin{cases}\frac{1}{2} & \text { if } p-q \text { is even } \\
1 & \text { if } p-q \text { is odd. }\end{cases}
\end{gathered}
$$

The baryon number is $B_{a}=2 \sigma_{p-a} m\left(p^{2}+q^{2}\right)$, while for the $g$-factor we get

$$
\begin{aligned}
g_{a}(p, q, m, \tau)= & L_{r} L_{\gamma} L_{\phi} \frac{K \pi^{3}}{4 \sigma_{p-q} m}\left[\frac{16 \sigma_{p-q}^{2}}{L_{\varphi}^{2}}+\frac{5}{\left(p^{2}+q^{2}\right) L_{r}^{2}}+\frac{\lambda \sigma_{p-q}^{2}}{L_{\varphi}^{2} L_{r}^{2}}+8 \frac{m^{2}}{L_{\gamma}^{2}}\left(1+\frac{\lambda}{16 L_{r}^{2}}\right)\right. \\
& \left.+8 \sigma_{p-q}^{2} \frac{m^{2}}{L_{\gamma}^{2}} \frac{\lambda\left(p^{2}+q^{2}\right)}{L_{\varphi}^{2}}\left(1-\frac{3 \tau^{2}}{p^{2}+q^{2}}+\frac{3 \tau^{4}}{\left(p^{2}+q^{2}\right)^{2}}+\frac{4 p^{2} q^{2}}{\left(p^{2}+q^{2}\right)^{2}}+\frac{3 p^{4} q^{4}}{\left(p^{2}+q^{2}\right)^{2}} \frac{1}{\tau^{4}}-\frac{3 p^{2} q^{2}}{p^{2}+q^{2}} \frac{1}{\tau^{2}}\right)\right] .
\end{aligned}
$$

The corresponding minimal energy, expressed in normalized units, is given by Eq. (G16), which in this case becomes

$$
g_{a}(p, q, \tau)=\frac{\pi}{3 \sqrt{2}}\left[2+\sqrt{5}\left(1-\frac{3 \tau^{2}}{p^{2}+q^{2}}+\frac{3 \tau^{4}}{\left(p^{2}+q^{2}\right)^{2}}+\frac{4 p^{2} q^{2}}{\left(p^{2}+q^{2}\right)^{2}}+\frac{3 p^{4} q^{4}}{\left(p^{2}+q^{2}\right)^{2}} \frac{1}{\tau^{4}}-\frac{3 p^{2} q^{2}}{p^{2}+q^{2}} \frac{1}{\tau^{2}}\right)^{\frac{1}{2}}\right] .
$$

We can further minimize with respect to $\tau$. Setting $x=\tau^{2}$, we have to find the stationary points in

$$
q^{2}<x<p^{2}
$$

Deriving the expression in the square root with respect to $x$ and multiplying by $\left(p^{2}+q^{2}\right)^{2} x^{3} / 6$, we get the equation

$$
0=\left(x^{2}-p^{2} q^{2}\right)\left(x^{2}-\frac{x}{2}\left(p^{2}+q^{2}\right)+p^{2} q^{2}\right) .
$$

This gives the admissible solutions ( $x$ is positive)

$$
x_{0}=p q, \quad x_{ \pm}=\frac{p^{2}+q^{2}}{4} \pm \sqrt{\frac{\left(p^{2}+q^{2}\right)^{2}}{16}-p^{2} q^{2}} .
$$

Note that $x_{0}$ is always present, while $x_{ \pm}$are stationary points only when the square root is real, that is, when

$$
\left(p^{2}+q^{2}\right)^{2}-16 p^{2} q^{2}>0 .
$$

Setting $z=p / q$ this means $x^{4}-14 x^{2}+1>0$ so (since $p / q>1)$

$$
x^{2}>7+\sqrt{48}=(2+\sqrt{3})^{2},
$$

and, finally,

$$
\frac{p}{q}>2+\sqrt{3} .
$$

Taking the second derivative of the above expression and evaluating it in $x_{0}$, we get that $x_{0}$ is the absolute minimum (at fixed $p$ and $q$ ) if

$$
9-\frac{p}{q}-\frac{q}{p}>0,
$$

that is (recalling $p \geq q$ ), for

$$
1 \leq \frac{p}{q}<\frac{1}{2}(9+\sqrt{77}),
$$


otherwise the minimum is placed in $x_{ \pm}$. In conclusion

$$
\begin{gathered}
g_{a, \min }(p, q)=\frac{\pi}{3 \sqrt{2}}\left[2+\sqrt{5} \chi_{a}(p, q)\right], \\
\chi_{a}(p, q)= \begin{cases}1+10 \frac{p^{2} q^{2}}{\left(p^{2}+q^{2}\right)^{2}}-6 \frac{p q}{p^{2}+q^{2}} & \text { if } 1<\frac{p}{q}<\frac{1}{2}(9+\sqrt{77}) \\
1-2 \frac{p^{2} q^{2}}{\left(p^{2}+q^{2}\right)^{2}} & \text { otherwise. }\end{cases}
\end{gathered}
$$

The absolute minimum in the family is the minimum of the first row. Setting $x=p q /\left(p^{2}+q^{2}\right)$, we see that $1+$ $10 x^{2}-6 x$ has a minimum for $x=3 / 10$, which corresponds to $p=3, q=1$. The corresponding absolute minimal energy is exactly Eq. (4.73). This is not surprising at all since $(p, q)=(3,1), \varepsilon=(1,1,1)$ correspond to solution (4.69) for $N=4$ [if we use Eq. (4.57) with $\Lambda=$ 2 in Eq. (E59)]. This corresponds to the undeformed $S U(2)$ embedding, as anticipated.

\section{The case $\varepsilon_{b}=(1,1,-1)$}

In this case we get

$$
v_{b}=i \operatorname{diag}(1,0,-1,0), \quad\left\|v_{b}\right\|^{2}=2 .
$$

Regarding the ranges, we can use the same exact reasoning as for the previous case, so we get

$U_{b}(t, \varphi, r, \gamma)=g_{4}\left(\sigma_{p-q} \Phi\right)\left(\begin{array}{cccc}e^{\frac{1}{2} i r} & 0 & 0 & 0 \\ 0 & 1 & 0 & 0 \\ 0 & 0 & e^{-\frac{1}{2} i r} & 0 \\ 0 & 0 & 0 & 1\end{array}\right) g_{4}(m \gamma)$,

$\Phi=\frac{t}{L_{\varphi}}-\varphi, \quad \sigma_{p-q}= \begin{cases}1 & \text { for } p-q \text { odd } \\ \frac{1}{2} & \text { for } p-q \text { even }\end{cases}$

The baryonic charge is

$$
B=2 \sigma_{p-q} m\left(p^{2}+q^{2}\right) .
$$

For the $g$-factor we get

$$
\begin{aligned}
g_{b}(p, q, m, \tau)= & L_{r} L_{\gamma} L_{\phi} \frac{K \pi^{3}}{4 \sigma_{p-q} m}\left[\frac{16 \sigma_{p-q}^{2}}{L_{\varphi}^{2}}+\frac{2}{\left(p^{2}+q^{2}\right) L_{r}^{2}}+\frac{\sigma_{p-q}^{2} \lambda}{L_{\varphi}^{2} L_{r}^{2}}+8 \frac{m^{2}}{L_{\gamma}^{2}}\left(1+\frac{\lambda}{16 L_{r}^{2}}\right)\right. \\
& \left.+8 \frac{m^{2}}{L_{\gamma}^{2}} \frac{\sigma_{p-q}^{2} \lambda\left(p^{2}+q^{2}\right)}{L_{\varphi}^{2}}\left(1+\frac{p^{2} q^{2}}{\left(p^{2}+q^{2}\right)^{2}}+\frac{3 \tau^{4}}{\left(p^{2}+q^{2}\right)^{2}}-\frac{3 \tau^{2}}{p^{2}+q^{2}}\right)\right] .
\end{aligned}
$$

The corresponding minimal energy, given by Eq. (G16), in this case becomes

$$
g_{b}(p, q, \tau)=\frac{\pi}{3 \sqrt{2}}\left[2+\sqrt{2}\left(1+\frac{p^{2} q^{2}}{\left(p^{2}+q^{2}\right)^{2}}+\frac{3 \tau^{4}}{\left(p^{2}+q^{2}\right)^{2}}-\frac{3 \tau^{2}}{p^{2}+q^{2}}\right)^{\frac{1}{2}}\right] .
$$

We can further minimize with respect to $\tau$. Setting $x=\tau^{2}$, it is immediate to see that in this case the minimum is reached for

$$
x_{0}=\frac{p^{2}+q^{2}}{2}
$$

corresponding to the value $g_{b, \min }(p, q)=\frac{\pi}{3 \sqrt{2}}\left[2+\sqrt{2}\left(\frac{1}{4}+\frac{p^{2} q^{2}}{\left(p^{2}+q^{2}\right)^{2}}\right)^{\frac{1}{2}}\right]$.

For fixed $q$, this is a monotonic decreasing function of $p$, so there is not an absolute minimum in this family. However, notice that the lower bound is 


$$
\begin{aligned}
g_{b, \text { bound }} & =\frac{\pi}{3 \sqrt{2}} \lim _{p \rightarrow \infty}\left[2+\sqrt{2}\left(\frac{1}{4}+\frac{p^{2} q^{2}}{\left(p^{2}+q^{2}\right)^{2}}\right)^{\frac{1}{2}}\right] \\
& =\frac{\pi}{6}(1+2 \sqrt{2}),
\end{aligned}
$$

which is Eq. (4.73).

We finally notice that these kinds of solutions are not deformations of an $S U(2)$ or $S O(3)$ embedding, despite what one might suspect. Indeed, $v_{b}$ may at most belong to the representation $8 \frac{1}{2} \oplus \mathbf{0} \oplus \mathbf{0}$ or $\mathbf{1} \oplus \mathbf{0}$ embedded in $S U(4)$. If so, there should exist a deformation of $k_{\underline{c}}$, that is a particular value of the moduli, such that $k_{\underline{c}}$ belongs to the same representation. But in both cases the particular solution would also be embedded in $S U(3)$, and then it would require $q=0$ or $p=0$.

\section{The case $\varepsilon_{c}=(1,-1,1)$}

In this case we have

$$
v_{c}=\frac{i}{2}(1,-1,1,-1), \quad\left\|v_{c}\right\|^{2}=1 .
$$

Reasoning as before, we see that the field is now

$$
\begin{gathered}
U_{c}=g_{4}\left(\sigma_{p-q} \Phi\right)\left(\begin{array}{cccc}
e^{\frac{1}{4} i r} & 0 & 0 & 0 \\
0 & e^{-\frac{1}{4} i r} & 0 & 0 \\
0 & 0 & e^{\frac{1}{4} i r} & 0 \\
0 & 0 & 0 & e^{-\frac{1}{4} i r}
\end{array}\right) g_{4}(m \gamma), \\
\Phi=\frac{t}{L_{\varphi}}-\varphi, \\
\sigma_{p-q}= \begin{cases}1 & \text { for } p-q \text { odd } \\
\frac{1}{2} & \text { for } p-q \text { even }\end{cases}
\end{gathered}
$$

The baryonic charge is

$$
B=2 \sigma_{p-q} m\left(p^{2}+q^{2}\right) .
$$

For the $g$-factor we get

$$
\begin{aligned}
g_{c}(p, q, m, \rho)= & L_{r} L_{\gamma} L_{\phi} \frac{K \pi^{3}}{\sigma_{p-q} m}\left[\frac{16 \sigma_{p-q}^{2}}{L_{\varphi}^{2}}+\frac{1}{2\left(p^{2}+q^{2}\right) L_{r}^{2}}+\frac{\lambda \sigma_{p-q}^{2}}{L_{\varphi}^{2} L_{r}^{2}}+8 \frac{m^{2}}{L_{\gamma}^{2}}\left(1+\frac{\lambda}{16 L_{r}^{2}}\right)\right. \\
& \left.+8 \sigma_{p-q}^{2} \frac{m^{2}}{L_{\gamma}^{2}} \frac{\lambda\left(p^{2}+q^{2}\right)}{L_{\varphi}^{2}}\left(1-\frac{2 p^{2} q^{2}}{\left(p^{2}+q^{2}\right)^{2}}\right)\right] .
\end{aligned}
$$

The corresponding minimal energy, given by Eq. (G16), in this case becomes

$g_{c}(p, q,|\rho|)=\frac{\pi}{3 \sqrt{2}}\left[2+\left(1-2 \frac{p^{2} q^{2}}{\left(p^{2}+q^{2}\right)^{2}}\right)^{\frac{1}{2}}\right]$.

This is independent on $\tau$, and for fixed $q$ it is a monotonic increasing function of $p$. It follows that the lower bound is reached for $p=q=1$ (the value 1 is enforced by the request that $p$ and $q$ are coprime, but the result depends only on $p / q$ ),

$$
g_{c, \text { bound }}=g_{c}(1,1)=\frac{\pi}{6}(1+2 \sqrt{2})
$$

which, again, is Eq. (4.73). However, this is not allowed since for $p=q=1$ the functions $f_{j}$ are not periodic and the solution of the equations does not yield a well-defined global solution. In this particular family the absolute minimum is instead

\footnotetext{
${ }^{8} \mathrm{We}$ are using the convention that $s$ indicates the representation of spin $s$.
}

$g_{c, \text { bound }}=g_{c}(2,1)=\frac{\pi}{3 \sqrt{2}}\left(2+\frac{\sqrt{17}}{5}\right) \simeq 2.0916$.

\section{The case $\varepsilon_{d}=(1,-1,-1)$}

In this case

$$
v_{d}=i(0,-1,0,1), \quad\left\|v_{d}\right\|^{2}=2 .
$$

This case seems to be very similar to the case $b$. Indeed, one can easily check that the matrices $v_{b}, k_{\underline{c}}$ transform into $v_{d}$, $k_{\underline{c}}$ under the map

$$
\begin{gathered}
\operatorname{Mat}(N, \mathbb{C}) \rightarrow \operatorname{Mat}(N, \mathbb{C}), \\
a_{j, k} \longmapsto a_{N-j, N-k}, \\
\mathbb{T}^{3} \times W \rightarrow \mathbb{T}^{3} \times W, \\
\left(e^{i \alpha_{1}}, e^{i \alpha_{2}}, e^{i \alpha_{3}}, \tau\right) \longmapsto\left(e^{i \alpha_{3}}, e^{i \alpha_{2}}, e^{i \alpha_{1}}, p q / \tau\right) .
\end{gathered}
$$

Under this map the inverse Cartan matrix is invariant and $\underline{\varepsilon}_{b} \mapsto-\underline{\varepsilon}_{d} \equiv \underline{\varepsilon}_{d}$, where the last equivalence is by a global rescaling. This sort of duality makes the two families perfectly equivalent, giving the same minima. 
Remark: We see that of the four predicted sequences of families, the true inequivalent ones are the first three, while the $d$ case is not really new. It is natural to expect that such duality extends to any $N$, but this would require a deeper understanding of the global properties of the relevant moduli space $W$. To this aim, it would be interesting to investigate the explicit cases $N=5$ and $N=6$. However, this goes beyond the scope of the present work.

\section{SHEAR MODULUS FOR LASAGNA STATES}

On the crust of ultracompact objects, like neutron stars, nucleons form large structures called pasta states. Knowing the elasticity properties of the crust may be very important to understand the structure of the gravitational waves emitted in a collision with a black hole. An important recent result has been found in Ref. [12] where, using numerical simulations based on the phenomenological nucleon-nucleon potential, the authors showed that the shear modulus for nuclear lasagna can have a value much larger than previous estimates. Here we give a firstprinciples explanation of it as an application of the skyrmionic model. To compute the shear modulus associated with lasagna states, our strategy will be to first compute it for the $S U(2)$ case for the solutions determined in Refs. [46,49], by employing its relation with the $1+1$ computations presented in Ref. [58].

Let us begin with a review [49]. We consider the symmetric case ${ }^{9}$ in Eqs. (13) and (16) of Ref. [49], namely,

$$
p=q, \quad l_{2}=l_{3}=\sqrt{A} .
$$

This means that we are considering configurations in which the $S U(2)$ skyrmions live in a box of volume $V_{\text {tot }}$,

$$
V_{\text {tot }}=16 \pi^{3} A l_{1}
$$

where $l_{1}$ is the length along the $r$ direction [which is the coordinate of the profile $H$ in Eq. (13) of Ref. [49]]. The baryonic charge corresponding to the ansatz in Eqs. (12)(14) of Ref. [49] is

$$
B=p q=p^{2}
$$

[see below Eq. (24) of Ref. [49]]. Then, the SU(2) field equations for the ansatz in Eqs. (12)-(14) and (16) of Ref. [49], with a static profile $H=H(r)$, reduce to

$$
-\frac{d^{2} u}{d r^{2}}+\Gamma^{2} \sin u=0
$$

where

\footnotetext{
${ }^{9}$ Notice that we are referring to the $p$ and $q$ in Ref. [49], which have different meaning than the $p$ and $q$ used in the previous section.
}

$$
\begin{gathered}
u(r)=4 H(r), \quad 0 \leq r \leq 2 \pi, \\
\Gamma^{2}=\left(\frac{B}{A}\right)^{2} \frac{\lambda l_{1}^{2}}{4+2 \lambda \frac{B}{A}},
\end{gathered}
$$

where $\frac{B}{A}$ can be interpreted as the baryon density per unit of area of the lasagna configuration (up to $\pi$ factors). In order to directly compare the present results with the ones in Ref. [58], it is convenient to define the rescaled coordinate $y$ as follows:

$$
y=\Gamma r, \quad 0 \leq y \leq 2 \pi \Gamma,
$$

so that the field equation (5.2) becomes

$$
-\frac{d^{2} u}{d y^{2}}+\sin u=0 \Leftrightarrow \frac{\left(\frac{d u}{d y}\right)^{2}}{2}=1-\cos u+C,
$$

and the boundary conditions, in order to have baryonic charge $B=p q=p^{2}$, are

$$
H(2 \pi)=\frac{\pi}{2} \Leftrightarrow u(2 \pi \Gamma)=2 \pi .
$$

Now, Eqs. (5.5)-(5.7) (which are equivalent to the results in Ref. [49]) can be compared directly with Eqs. (2.4), (2.7), and (2.9) of Ref. [58]. In particular, the difference between the results of Ref. [58] and the present ones is

$$
\begin{gathered}
\phi(x) \rightarrow u(y), \\
L \rightarrow 2 \pi \Gamma, \\
k \rightarrow \sqrt{\frac{2}{C+2}} \equiv \tau, \\
k^{\prime} \rightarrow \sqrt{\frac{C}{C+2}}
\end{gathered}
$$

where the left-hand side (with respect to the " $\rightarrow$ ") is from Ref. [58] while the right-hand side comes from the above equations. Equations (2.9) and (2.10) of Ref. [58] read

$$
\begin{gathered}
L=2 \tau I_{-1 / 2}(\tau), \\
I_{-1 / 2}(\tau)=\int_{0}^{\pi / 2} d y\left(1-\tau^{2} \sin ^{2} y\right)^{-1 / 2},
\end{gathered}
$$

that is,

$\pi \Gamma=\sqrt{\frac{2}{C+2}} \int_{0}^{\pi / 2} d y\left(1-\frac{2}{C+2} \sin ^{2} y\right)^{-1 / 2}$,

which fixes the integration constant $C$ in Eq. (5.6) in terms of $\Gamma$, 


$$
C=C(\Gamma),
$$

which depends on the baryon charge as well as the size of the box in which the configuration lives. Now, with the above dictionary, we can write the speed of sound of the phonons using Eq. (3.15) of Ref. [58]:

$$
\begin{aligned}
V_{\text {phonons }} & =\sqrt{\frac{C}{2}} \frac{\pi \Gamma}{\int_{0}^{\pi / 2} d y\left(1-\frac{2}{C+2} \sin ^{2} y\right)^{1 / 2}} \\
& =\sqrt{\frac{G_{S U(2)}}{T_{00}}},
\end{aligned}
$$

where $T_{00}$ is given in Eq. (28) of Ref. [49]. Thus, we have the following expression for the shear modulus $G_{S U(2)}$ in the $S U(2)$ case:

$$
G_{S U(2)}=\left(V_{\text {phonons }}\right)^{2} T_{00} .
$$

We can then estimate it as follows. In place of $T_{00}$ we use its mean value, computed as

$$
\bar{T}_{00}=\frac{E_{\min }^{0} B}{16 \pi^{3} l_{1} A},
$$

where $E_{\min }^{0}$ is the minimal energy corresponding to $B=1$. From Table 1 of Ref. [58], we see that $B / A$ is independent from $B$ for the minimal energy configuration. Using the values in the table, ${ }^{10}$ we get

$$
\bar{T}_{00} \simeq 1.2610^{34} \mathrm{erg} / \mathrm{cm}^{3} .
$$

With the same values, from Eq. (5.4) we obtain

$$
\Gamma \simeq 0.371, \quad \pi \Gamma \simeq 1.166 .
$$

Therefore, condition (5.12), which is easily solved numerically after noticing that $I_{-\frac{1}{2}}(\tau)=K\left(\tau^{2}\right)$, the first complete elliptic integral, gives

$$
C \simeq 2.73
$$

and

$$
V_{\text {phonons }} \simeq 0.1198
$$

Finally,

$$
G_{S U(2)} \simeq 1.810^{32} \mathrm{erg} / \mathrm{cm}^{3}
$$

\footnotetext{
${ }^{10}$ Notice that with these values the baryon density is $n \simeq 0.0468 \mathrm{fm}^{-3} \approx 0.05 \mathrm{fm}^{-3}$, the same value used in the simulations of Ref. [12].
}

Notice that the present value is expected, to an approximation, from above since we are using a skyrmionic effective model. From the above analysis, taking into account Eq. (4.73), we can infer that, in any case, the true value should be $G_{S U(2)} \gtrsim 10^{31} \mathrm{erg} / \mathrm{cm}^{3}$. The comparison with Ref. [12] is very good, especially taking into account that we only used the Skyrme model.

At this point we can use the new solutions found in the present work to relate the shear modulus for the $S U(N)$ case to the one for $S U(2)$.

Let us consider the minimal energy per nucleon (4.72). After multiplying by $B$ and dividing by the volume, which, because of (IV C) is proportional to $\lambda^{\frac{3}{2}}$, we get

$$
\bar{T}_{00} \propto \frac{K}{\lambda} N\left(N^{2}-1\right) .
$$

On the other hand, the baryon density is

$$
n=\frac{B}{8 \pi^{2} L_{\varphi} L_{r} L_{\gamma}} \propto \frac{N\left(N^{2}-1\right)}{\lambda^{3 / 2}},
$$

which, when solved for $\lambda$ and replaced in $\bar{T}_{00}$, gives

$$
\bar{T}_{00} \propto n^{2 / 3} \sqrt[3]{N\left(N^{2}-1\right)}
$$

Assuming the speed of sound to be essentially independent from $N$, as suggested by the fact that all the components of $T_{\mu \nu}$ scale in the same way with $N$, we get that the dependence of the shear modulus from $N$ is

$$
G_{S U(N)} \propto \sqrt[3]{N\left(N^{2}-1\right)},
$$

so we get the final estimate for the value of the shear modulus $G_{S U(N)}$ of the $S U(N)$ Skyrme model as

$$
\begin{aligned}
G_{S U(N)} & =a(N) G_{S U(2)}, \\
a(N) & =\sqrt[3]{\frac{N\left(N^{2}-1\right)}{6}} .
\end{aligned}
$$

\section{CONCLUSION AND PERSPECTIVES}

In conclusion, we constructed the first examples of analytic $(3+1)$-dimensional skyrmions living at finite baryon density in the SU(N) Skyrme model [which are not trivial embeddings of $S U(2)$ into $S U(N)$ ] for any $\mathrm{N}$. These results allow us to explicitly compute the energy-tobaryon charge ratio for any $N$ and to discuss its smooth large- $N$ limit as well as the closeness to the BPS bound. The energy density profiles of these finite density skyrmions have a lasagna-like shape. A quite remarkable byproduct of the present analysis is that we have been able to analytically estimate the shear modulus of lasagna-shaped 
configurations which appear at finite baryon density. Our estimate agrees with recent results [12] based on manybody simulations in nuclear physics using phenomenological nucleon-nucleon interaction potentials.

\section{ACKNOWLEDGMENTS}

This work has been funded by Fondecyt Grant No. 1200022, MINEDUC-UA Project No. ANT 1755 and by Semillero de Investigación SEM 18-02 of the Universidad de Antogasta. Centro de Estudios Científicos (CECs) is funded by the Chilean Government through the Centers of Excellence Base Financing Program of Conicyt.

\section{APPENDIX A: GENERAL FACTS AND CONVENTIONS ABOUT $S U(N)$}

In this section we collect some general facts we applied for finding the solutions. Let $V,(\mid)$ the $N$-dimensional complex vector space isomorphic to $\mathbb{C}^{N}$, endowed with the canonical Hermitian product

$$
\begin{aligned}
(\underline{z} \mid \underline{w}) & =\sum_{j=1}^{n} z_{j}^{*} w_{j}, \quad z, w \in \mathbb{C}^{n}, \\
(x+i y)^{*} & =x-i y, \quad x, y \in \mathbb{R} .
\end{aligned}
$$

The unitary group $U(N) \equiv U(V)$ is the group of unitary transformations of $V$. Looking at $U(V)$ as automorphisms of $V$ determines the smallest fundamental representation, simply called $V$. The action of $U(V)$ over $V$ induces an action on the external products $\wedge^{k} V$ of $V$, and the corresponding homomorphisms

$$
U(V) \rightarrow \operatorname{Aut}\left(\wedge^{k} V\right), \quad k=1, \ldots, N
$$

are all representations, also called $\wedge^{k} V$. For $k=1, \ldots$, $N-1$ are all faithful (that is the kernel of the map is the identity transformation) and are called the fundamental representations. Any other finite-dimensional representation is obtained by their tensor products. Note that $\wedge^{N} V$ is not faithful. The corresponding kernel is a normal subgroup of $U(N)$ called the special group $S U(N) \equiv S U(V)$.

Here, $S U(N)$ is a compact, simply connected, simple Lie group of rank $N-1$. This essentially means that it contains a maximal Abelian torus $T_{N}$ of dimension $N-1$. On $V$, it is represented by the diagonal $N \times N$ matrices $T$ such that

$$
\prod_{j=1}^{N} T_{j j}=1, \quad\left|T_{j j}\right|=1, \quad j=1, \ldots, N .
$$

The center $Z_{N}$ of $S U(N)$ is the subgroup of $T$ consisting of the elements commuting with the whole $S U(N)$ (equivalently, it is the kernel of the adjoint representation). It consists of matrices of the form $\omega \mathbb{I}$, where $\omega^{N}=1$ and $\mathbb{I}$ is the identity matrix. Therefore, $Z_{N} \simeq \mathbb{Z}_{N}$. All of the other compact simple Lie groups locally isomorphic to $S U(N)$ are the quotients $S U(N)_{\Gamma}:=S U(N) / \Gamma$, where $\Gamma$ is any given subgroup of $Z_{N}$. They are not simply connected since their first homotopy group is $\pi_{1}\left(S U(N)_{\Gamma}\right)=Z_{N} / \Gamma$. Note that $S U(N)$ is the universal covering for all of them. In particular, for $N=2$ we have just two groups, which are $S U(2)$ and $S U(2)_{\mathbb{Z}_{2}} \simeq S O(3)$.

To any Lie group $G$, one associates the corresponding Lie algebra $L(G)$, which is the algebra of left-invariant vector fields ${ }^{11}$ over $G$, endowed with the Lie bracket product. In matrix representation it reduces to the commutator [, ]. Since the groups $S U(N)_{\Gamma}$ are locally isomorphic to $S U(N)$, their Lie algebras are all isomorphic. One gets

$$
\begin{aligned}
\mathfrak{g} \mathfrak{t}(N) & \equiv \operatorname{Lie}(\operatorname{SU}(N)) \\
& =\left\{X \in \operatorname{Mat}(N) \mid X^{\dagger}=-X, \operatorname{Tr} X=0\right\},
\end{aligned}
$$

that is the anti-Hermitian traceless $N \times N$ complex matrices.

In particular, $H:=\operatorname{Lie}\left(T_{N}\right)$ is a maximal Abelian subalgebra of $\mathfrak{g} \mathfrak{u}(N)$, having the property that, for any $X \in H$, the linear map $a d_{X}: \mathfrak{s} \mathfrak{u}(N) \rightarrow \mathfrak{s} \mathfrak{u}(N)$ defined by ${ }^{12}$ $\operatorname{ad}_{X}(Y)=[X, Y]$ for any $Y \in \mathfrak{s} \mathfrak{t}(N)$ is diagonalizable (on the complexification of the algebra).

We see from the definition that $\mathfrak{g} \mathfrak{u}(N)$ is a real vector space of dimension $N^{2}-1$. A basis can be easily obtained as follows. For any $j, k=1, \ldots, N$ we define the matrix $E_{j, k}$ with elements

$$
\left(E_{j, k}\right)_{m n}=\delta_{j m} \delta_{k n} .
$$

They are called the elementary matrices. With these notations, a basis of $\mathfrak{g} \mathfrak{u}(N)$ is given by

$$
\begin{aligned}
& A_{j, k}=\left(E_{j, k}-E_{k, j}\right), \quad S_{j, k}=i\left(E_{j, k}+E_{k, j}\right), \\
& 1 \leq j<k \leq N, \\
& \quad J_{h}=i\left(E_{j, j}-E_{j+1, j+1}\right), \quad h=1, \ldots, N-1 .
\end{aligned}
$$

In particular, the matrices $J_{h}$ span the Cartan subalgebra $H$.

\section{Roots and simple roots}

A concept that is particularly helpful for most of the calculations we need is the one of roots. This is related to the above observation regarding the diagonalizability of $a d_{X}$ for any $X \in H$. The diagonalizability must be checked on $\mathfrak{s} \mathfrak{t}(N) \otimes \mathbb{C}$, which is generated by the complex span of the basis given above, in place of the real span. Notice that

\footnotetext{
${ }^{11}$ That is the vector fields invariant under the action of the left translation $L_{g}: G \rightarrow G, L_{g}(h)=g h$, for any given $g \in G$.

${ }^{12}$ This is called the adjoint action, and it defines the adjoint representation of the algebra over itself.
} 
the complex span contains the matrices $E_{i, j}, i \neq j$. This is sufficient to determine all the eigenvectors and eigenvalues of $a d_{X}$ for any given $X \in H$.

To this aim, let us specify $H$ as follows:

$$
H=\left\langle X=i \sum_{j=1}^{n} c_{j} E_{j, j} \mid \sum_{i} c_{i}=0\right\rangle_{\mathbb{R}},
$$

where by $\langle\cdots\rangle_{\mathbb{R}}$ we mean the span over $\mathbb{R}$ of $\cdots$. Thus, we immediately see that

$$
\begin{gathered}
{\left[X, E_{j, k}\right]=i\left(c_{j}-c_{k}\right) E_{j, k},} \\
{\left[X, J_{h}\right]=0,}
\end{gathered}
$$

so that $E_{j, k}$ and $J_{h}$ are eigenmatrices of the adjoint action of $X$, with eigenvalues $i\left(c_{j}-c_{k}\right)$ and 0 , respectively. The point is that the eigenvalues depend linearly on $X$. Let us consider the linear operators $L_{j}, j=1, \ldots, N$ defined by

$$
L_{j}: \operatorname{Mat}(N) \rightarrow \mathbb{C}, \quad A \mapsto A_{j, j} .
$$

Then, we can write $i c_{j}=L_{j}(X)$ so that

$$
a d_{X}\left(E_{j, k}\right)=\left(L_{j}-L_{k}\right)(X) E_{j, k} .
$$

The linear operators

$$
\beta_{i, j}:=L_{j}-L_{k}: H \rightarrow \mathbb{C}
$$

are said to be the nonvanishing roots of $\mathfrak{g} \mathfrak{u}(N)$. The corresponding eigenspaces are one dimensional. Beyond these, there is a vanishing root defining the 0 eigenvalue, whose eigenspace is $H$, so it has degeneration equal to the rank $r=N-1$.

In particular, the set of nonvanishing roots contains a set of $r$ linearly independent roots, having the property that all the remaining roots are a combination of them, with all nonpositive or all non-negative integer coefficients. These are called the simple roots, and they are

$$
\beta_{j}:=L_{j}-L_{j+1}, \quad j=1, \ldots, N-1 .
$$

Finally, for convenience, we introduce the less conventional concept of real valued roots $\alpha_{j, k}=-i \beta_{j, k}, \alpha_{j}=-i \beta_{j}$, which we simply call roots and simple roots. With this convention, for the simple roots $\alpha_{j}$, we can also write

$$
\alpha_{j}: H \rightarrow \mathbb{R}, \quad X \mapsto-\operatorname{Tr}\left(J_{j} X\right),
$$

which is useful for practical purposes. This also shows that the $\alpha_{j}$ are linearly independent. We name the corresponding eigenvectors $\lambda_{\alpha_{j}} \equiv \lambda_{j}=E_{j, j+1}$, so that

$$
\left[X, \lambda_{j}\right]=i \alpha_{j}(X) \lambda_{j}, \quad \forall X \in H .
$$

Notice that $\lambda_{-\alpha_{j}}=\lambda_{\alpha_{j}}^{\dagger}$, so

$$
\left[X, \lambda_{j}^{\dagger}\right]=-i \alpha(X) \lambda_{j}^{\dagger} .
$$

\section{Some further technical facts}

There is a canonical way to introduce a scalar product on the real space spanned by the simple roots. However, we bypass the historical construction and employ Eq. (A10) to define the scalar product

$$
\left(\alpha_{j} \mid \alpha_{k}\right):=-\operatorname{Tr}\left(J_{j} J_{k}\right) .
$$

On

$$
H_{\mathbb{R}}^{*}:=\left\langle\alpha_{1}, \ldots, \alpha_{N-1}\right\rangle_{\mathbb{R}}
$$

it is a Euclidean scalar product. One then defines the $r \times r$ Cartan matrix ${ }^{13} C_{A_{N-1}}$ with components

$$
\begin{aligned}
\left(C_{A_{N-1}}\right)_{j, k} & :=2 \frac{\left(\alpha_{j} \mid \alpha_{k}\right)}{\left(\alpha_{j} \mid \alpha_{j}\right)}=\left(\alpha_{j} \mid \alpha_{k}\right) \\
& =2 \delta_{j, k}-\delta_{j, k+1}-\delta_{j, k-1} .
\end{aligned}
$$

The Cartan matrix is strictly positive definite. Indeed, for any vector $\left(x^{1}, \ldots, x^{r}\right) \in \mathbb{R}^{r}$, we have

$$
\begin{aligned}
\sum_{j, k} x^{j} x^{k}\left(C_{A_{N-1}}\right)_{j, k} & =2 \sum_{j=1}^{r} x_{j}^{2}-\sum_{j=1}^{r-1} 2 x_{j} x_{j+1} \\
& =x_{1}^{2}+x_{r}^{2}+\sum_{j=1}^{r-1}\left(x_{j}-x_{j+1}\right)^{2}
\end{aligned}
$$

which is strictly positive and vanishes only for $x_{j}=0$ for all $j$. In particular, the Cartan matrix is invertible and, indeed, one can easily check that

$$
\left(C_{A_{N-1}}^{-1}\right)_{j, k}=\frac{1}{N} \min (j, k)(N-\max (j, k)) .
$$

Another important fact to notice is that for $j, k$ one has

$$
\left[\lambda_{j}, \lambda_{k}\right]=\delta_{j+1, k} E_{j, j+2} .
$$

\section{APPENDIX B: PROOF OF PROPOSITION 1}

In order to prove the proposition, it is convenient to work with the coordinates $\Phi$ and $T=t+L_{\varphi} \varphi$. The metric takes the form $d s^{2}=-L_{\varphi} d T d \Phi+L_{r}^{2} d r^{2}+L_{\gamma}^{2} d \gamma^{2}$. With the given ansatz, after replacing $\Phi$ with $\sigma \Phi$ for constant $\sigma$ (for convenience), for the $L_{\mu}$ we get

\footnotetext{
${ }^{13}$ The name comes from the fact that in the Dynkin classification, the algebra $s u(N)$ is called $A_{r}$, where $r$ is the rank.
} 


$$
\begin{aligned}
R_{T} & =0, & R^{T} & =-\frac{2}{L_{\varphi}} R_{\Phi}, \\
R_{\Phi} & =\sigma e^{-m \gamma k} e^{-h(r)} k e^{h(r)} e^{m \gamma k}, & R^{\Phi} & =0, \\
R_{r} & =e^{-m \gamma k} h^{\prime}(r) e^{m \gamma k}, & R^{r} & =\frac{1}{L_{r}^{2}} R_{r}, \\
R_{\gamma} & =m k, & R^{\gamma} & =\frac{1}{L_{\gamma}^{2}} R_{\gamma} .
\end{aligned}
$$

For $F_{\mu \nu}=\left[R_{\mu}, R_{\nu}\right]$, with $x=e^{-h(r)} k e^{h(r)}$, we get the nonvanishing components

$$
\begin{aligned}
F_{\Phi r} & =-F_{r \Phi}=\sigma e^{-m \gamma k}\left[x, h^{\prime}\right] e^{m \gamma k}, \\
F_{\Phi \gamma} & =-F_{\gamma \Phi}=\sigma m e^{-m \gamma k}[x, k] e^{m \gamma k}, \\
F_{r \gamma} & =-F_{\gamma r}=m e^{-m \gamma k}\left[h^{\prime}, k\right] e^{m \gamma k} .
\end{aligned}
$$

Setting $\mathcal{L}_{\mu}:=\left[L^{\nu}, F_{\mu \nu}\right]$, the equations of motion are

$$
0=\partial^{\mu} R_{\mu}+\frac{\lambda}{4} \partial^{\mu} \mathcal{L}_{\mu}
$$

Using the fact that nothing depends on $T$ and that there are no lower $T$ components, these reduce to

$$
0=\frac{1}{L_{r}^{2}} \partial_{r}\left(R_{r}+\frac{\lambda}{4} \mathcal{L}_{r}\right)+\frac{1}{L_{\gamma}^{2}} \partial_{\gamma}\left(R_{\gamma}+\frac{\lambda}{4} \mathcal{L}_{\gamma}\right) .
$$

But

$$
\begin{aligned}
\partial_{\gamma} R_{\gamma} & =0, \\
\partial_{r} R_{r} & =e^{-m \gamma k} h^{\prime \prime} e^{m \gamma k}, \\
\partial_{r} \mathcal{L}_{r} & =\partial_{r}\left(\frac{m^{2}}{L_{\gamma}^{2}} e^{-m \gamma k}\left[k,\left[h^{\prime}, k\right]\right] e^{m \gamma k}\right) \\
& =\frac{m^{2}}{L_{\gamma}^{2}} e^{-m \gamma k}\left[k,\left[h^{\prime \prime}, k\right]\right] e^{m \gamma k}, \\
\partial_{\gamma} \mathcal{L}_{\gamma} & =-\frac{m}{L_{r}^{2}} \partial_{\gamma}\left(e^{-m \gamma k}\left[h^{\prime},\left[h^{\prime}, k\right]\right] e^{m \gamma k}\right) \\
& =\frac{m^{2}}{L_{r}^{2}} e^{-m \gamma k}\left[k,\left[h^{\prime},\left[h^{\prime}, k\right]\right]\right] e^{m \gamma k},
\end{aligned}
$$

so Eq. (B1) becomes

$$
0=\frac{1}{L_{r}^{2}} e^{-m \gamma k}\left(h^{\prime \prime}-\frac{\lambda}{4} \frac{m^{2}}{L_{\gamma}^{2}}\left(\left[k,\left[k, h^{\prime \prime}\right]\right]-\left[k,\left[h^{\prime},\left[h^{\prime}, k\right]\right]\right]\right)\right) e^{m \gamma k}
$$

which proves the proposition.

\section{Further details}

Making use of Eqs. (3.6) and (A11), we can write

$$
\left[h^{\prime}, k\right]=\sum_{j=1}^{N-1}\left(c_{j}\left[h^{\prime}, \lambda_{j}\right]-c_{j}^{*}\left[h^{\prime}, \lambda_{j}^{\dagger}\right]\right)=i \sum_{j=1}^{N-1} \alpha_{j}\left(h^{\prime}\right)\left(c_{j} \lambda_{j}+c_{j}^{*} \lambda_{j}^{\dagger}\right) .
$$

Repeating the same calculation, we get

$$
\left[h^{\prime},\left[h^{\prime}, k\right]\right]=i \sum_{j=1}^{N-1}\left(\alpha_{j}\left(h^{\prime}\right) c_{j}\left[h^{\prime}, \lambda_{j}\right]+\alpha_{j}\left(h^{\prime}\right) c_{j}^{*}\left[h^{\prime}, \lambda_{j}^{\dagger}\right]\right)=-\sum_{j=1}^{N-1} \alpha_{j}\left(h^{\prime}\right)^{2}\left(c_{j} \lambda_{j}-c_{j}^{*} \lambda_{j}^{\dagger}\right) .
$$

Finally,

$$
\begin{aligned}
{\left[k,\left[h^{\prime},\left[h^{\prime}, k\right]\right]\right] } & =-\sum_{k=1}^{N-1} \sum_{j=1}^{N-1} \alpha_{j}\left(h^{\prime}\right)^{2}\left\{c_{k} c_{j}\left[\lambda_{k}, \lambda_{j}\right]+c_{k}^{*} c_{j}^{*}\left[\lambda_{k}^{\dagger}, \lambda_{j}^{\dagger}\right]-c_{k} c_{j}^{*}\left[\lambda_{k}, \lambda_{j}^{\dagger}\right]-c_{k}^{*} c_{j}\left[\lambda_{k}^{\dagger}, \lambda_{j}\right]\right\} \\
& =-\sum_{k=1}^{N-1} \sum_{j=1}^{N-1} \alpha_{j}\left(h^{\prime}\right)^{2}\left\{c_{k} c_{j}\left[\lambda_{k}, \lambda_{j}\right]+c_{k}^{*} c_{j}^{*}\left[\lambda_{k}^{\dagger}, \lambda_{j}^{\dagger}\right]-c_{k} c_{j}^{*}\left[\lambda_{k}, \lambda_{j}^{\dagger}\right]+c_{k}^{*} c_{j}\left[\lambda_{j}, \lambda_{k}^{\dagger}\right]\right\}
\end{aligned}
$$

The last two terms cancel after summation, while the first terms vanish for $j=k$; thus, we get

$$
\begin{aligned}
{\left[k,\left[h^{\prime},\left[h^{\prime}, k\right]\right]\right] } & =-\sum_{j<k} \alpha_{j}\left(h^{\prime}\right)^{2}\left(c_{j} c_{k}\left[\lambda_{k}, \lambda_{j}\right]+c_{j}^{*} c^{*} k\left[\lambda_{k}^{\dagger}, \lambda_{j}^{\dagger}\right]\right)-\sum_{k<j} \alpha_{j}\left(h^{\prime}\right)^{2}\left(c_{j} c_{k}\left[\lambda_{k}, \lambda_{j}\right]+c_{j}^{*} c^{*} k\left[\lambda_{k}^{\dagger}, \lambda_{j}^{\dagger}\right]\right) \\
& =\sum_{j<k}\left(\alpha_{j}\left(h^{\prime}\right)^{2}-\alpha_{k}\left(h^{\prime}\right)^{2}\right)\left(c_{j} c_{k}\left[\lambda_{j}, \lambda_{k}\right]+c_{j}^{*} c^{*} k\left[\lambda_{j}^{\dagger}, \lambda_{k}^{\dagger}\right]\right),
\end{aligned}
$$


where we have changed the order of commutators in the first sum and exchanged the name of the variable in the second sum. Therefore,

$$
\left[k,\left[h^{\prime},\left[h^{\prime}, k\right]\right]\right]=\sum_{j<k}\left(\alpha_{j}\left(h^{\prime}\right)^{2}-\alpha_{k}\left(h^{\prime}\right)^{2}\right)\left(c_{j} c_{k}\left[\lambda_{j}, \lambda_{k}\right]+c_{j}^{*} c_{k}^{*}\left[\lambda_{j}^{\dagger}, \lambda_{k}^{\dagger}\right]\right) .
$$

Similarly,

$$
\left[k, h^{\prime \prime}\right]=-\left[h^{\prime \prime}, k\right]=-i \sum_{j=1}^{N-1} \alpha_{j}\left(h^{\prime \prime}\right)\left(c_{j} \lambda_{j}+c_{j}^{*} \lambda_{j}^{\dagger}\right)
$$

and

$$
\left[k,\left[k, h^{\prime \prime}\right]\right]=-i \sum_{j=1}^{N-1} \sum_{k=1}^{N-1} \alpha_{j}\left(h^{\prime \prime}\right)\left[c_{j} c_{k}\left[\lambda_{k}, \lambda_{j}\right]-c_{j}^{*} c_{k}^{*}\left[\lambda_{k}^{\dagger}, \lambda_{j}^{\dagger}\right]-c_{j} c_{k}^{*}\left[\lambda_{k}^{\dagger}, \lambda_{j}\right]+c_{j}^{*} c_{k}\left[\lambda_{k}, \lambda_{j}^{\dagger}\right]\right]
$$

The first two terms can be treated as above, giving the contribution

$$
i \sum_{j<k}\left(\alpha_{j}\left(h^{\prime \prime}\right)-\alpha_{k}\left(h^{\prime \prime}\right)\right)\left(c_{j} c_{k}\left[\lambda_{j}, \lambda_{k}\right]-c_{j}^{*} c_{k}^{*}\left[\lambda_{j}^{\dagger}, \lambda_{k}^{\dagger}\right]\right),
$$

while the last two terms, after renaming the labels in the first of the sums, give the contribution

$$
-i \sum_{j=1}^{N-1} \sum_{k-1}^{N-1}\left(\alpha_{j}\left(h^{\prime \prime}\right)+\alpha_{k}\left(h^{\prime \prime}\right)\right) c_{k} c_{j}^{*}\left[\lambda_{k}, \lambda_{j}^{\dagger}\right] .
$$

Now,

$$
\left[\lambda_{k}, \lambda_{j}^{\dagger}\right]=\left[E_{k, k+1}, E_{j+1, j}\right]
$$

which in components is

$$
\left[E_{k, k+1}, E_{j+1, j}\right]_{m, r}=\delta_{j, k}\left(E_{j, j}-E_{j+1, j+1}\right)_{m, r}
$$

so that

$$
\left[\lambda_{k}, \lambda_{j}^{\dagger}\right]=-i \delta_{j, k} J_{j}
$$

We finally get

$$
\begin{gathered}
{\left[k,\left[k, h^{\prime \prime}\right]\right]=i \sum_{j<k}\left(\alpha_{j}\left(h^{\prime \prime}\right)-\alpha_{k}\left(h^{\prime \prime}\right)\right)} \\
\left(c_{j} c_{k}\left[\lambda_{j}, \lambda_{k}\right]-c_{j}^{*} c_{k}^{*}\left[\lambda_{j}^{\dagger}, \lambda_{k}^{\dagger}\right]\right)-2 \sum_{j=1}^{N-1} \alpha_{j}\left(h^{\prime \prime}\right)\left|c_{j}\right|^{2} J_{j} .
\end{gathered}
$$

\section{A further proposition}

We now want to state another technical proposition:

Proposition 5: Assume $k_{\underline{c}}=\sum_{j=1}^{N-1}\left(c_{j} E_{j, j+1}-c_{j}^{*} E_{j+1, j}\right)$, $h^{\prime} \in H$ a matrix such that $\alpha_{j}\left(h^{\prime}\right)=: \varepsilon_{j} a$ where $\varepsilon_{j}$ is a sign, $j=1, \ldots, N-1$, and $x:=e^{-h^{\prime} r} k_{\underline{c}} e^{h^{\prime} r}$. Then

$$
\operatorname{Tr} k_{\underline{c}}^{2}=-2\|\underline{c}\|^{2},
$$

$\operatorname{Tr}\left(\left[h^{\prime}, k_{\underline{c}}\right]\left[h^{\prime}, k_{\underline{c}}\right]\right)=\operatorname{Tr}\left(\left[h^{\prime}, x\right]\left[h^{\prime}, x\right]\right)=-2 a^{2}\|\underline{c}\|^{2}$,

and

$\operatorname{Tr}\left(\left[x, k_{\underline{c}}\right]\left[x, k_{\underline{c}}\right]\right)=-8 \sin ^{2}($ ar $)$

$$
\left(\sum_{j=1}^{N-1}\left|c_{j}\right|^{4}+\sum_{j=1}^{N-2}\left|c_{j}\right|^{2}\left|c_{j+1}\right|^{2} \frac{1}{2}\left(1-3 \varepsilon_{j} \varepsilon_{j+1}\right)\right) .
$$

Proof.-First, we have

$$
\begin{aligned}
\operatorname{Tr} k_{\underline{c}}^{2}= & \sum_{j=1}^{N-1} \sum_{k=1}^{N-1}\left\{c_{j} c_{k} \operatorname{Tr}\left(\lambda_{j} \lambda_{k}\right)+c_{j}^{*} c_{k}^{*} \operatorname{Tr}\left(\lambda_{j}^{\dagger} \lambda_{k}^{\dagger}\right)\right. \\
& \left.-c_{j}^{*} c_{k} \operatorname{Tr}\left(\lambda_{j}^{\dagger} \lambda_{k}\right)-c_{j} c_{k}^{*} \operatorname{Tr}\left(\lambda_{j} \lambda_{k}^{\dagger}\right)\right\},
\end{aligned}
$$

where we used the notation $\lambda_{j}=E_{j, j+1}$. Since $\lambda_{j}$ is upper diagonal so is $\lambda_{j} \lambda_{k}$; hence $\operatorname{Tr}\left(\lambda_{j} \lambda_{k}\right)=0$. Similarly, $\operatorname{Tr}\left(\lambda_{j}^{\dagger} \lambda_{k}^{\dagger}\right)=0$. On the other hand,

$$
\begin{aligned}
\operatorname{Tr}\left(\lambda_{j}^{\dagger} \lambda_{k}\right) & =\sum_{n=1}^{N} \sum_{m=1}^{N}\left(E_{j+1, j}\right)_{n m}\left(E_{k, k+1}\right)_{m n} \\
& =\sum_{n=1}^{N} \sum_{m=1}^{N} \delta_{j+1, n} \delta_{j m} \delta_{k+1, n} \delta_{k m} \\
& =\delta_{k j}=\operatorname{Tr}\left(\lambda_{j} \lambda_{k}^{\dagger}\right) .
\end{aligned}
$$

This proves Eq. (B6).

Now, notice that

$$
\left[h^{\prime}, x\right]=\left[h^{\prime}, e^{-h^{\prime} r} k_{\underline{c}} e^{h^{\prime} r}\right]=e^{-h^{\prime} r}\left[h^{\prime}, k_{\underline{c}}\right] e^{h^{\prime} r}
$$

since $h^{\prime}$ commutes with $e^{h^{\prime} r}$. Therefore, 


$$
\begin{aligned}
\operatorname{Tr}\left(\left[h^{\prime}, x\right]\left[h^{\prime}, x\right]\right) & =\operatorname{Tr}\left(e^{-h^{\prime} r}\left[h^{\prime}, k_{\underline{c}}\right]\left[h^{\prime}, k_{\underline{c}}\right] e^{h^{\prime} r}\right) \\
& =\operatorname{Tr}\left(\left[h^{\prime}, k_{\underline{c}}\right]\left[h^{\prime}, k_{\underline{c}}\right]\right)
\end{aligned}
$$

because of the cyclicity property of the trace. So we are left with the computation of $\operatorname{Tr}\left(\left[h^{\prime}, k_{c}\right]\left[h^{\prime}, k_{c}\right]\right)$. Using Eq. (B2) and the fact that the only nonvanishing traces are $\operatorname{Tr}\left(\lambda_{j}^{\dagger} \lambda_{k}\right)=\delta_{j, k}$, we get

$$
\begin{aligned}
\operatorname{Tr}\left(\left[h^{\prime}, k_{\underline{c}}\right]\left[h^{\prime}, k_{\underline{c}}\right]\right) & =\operatorname{Tr}\left(\sum_{j=1}^{N-1}\left(i \alpha_{j}\left(h^{\prime}\right) \lambda_{j} c_{j}+i \alpha_{j}\left(h^{\prime}\right) c_{j}^{*} \lambda_{j}^{\dagger}\right) \sum_{k=1}^{N-1}\left(i \alpha_{k}\left(h^{\prime}\right) \lambda_{k} c_{k}+i \alpha_{k}\left(h^{\prime}\right) c_{k}^{*} \lambda_{k}^{\dagger}\right)\right) \\
& =-2 \sum_{j=1}^{N-1} \alpha_{j}\left(h^{\prime}\right)^{2} c_{j} c_{j}^{*}=-2 a^{2}\|\underline{c}\|^{2},
\end{aligned}
$$

where we used $\alpha_{j}\left(h^{\prime}\right)^{2}=\left(\varepsilon_{j} a\right)^{2}=a^{2}$. This proves Eq. (B7).

Let us write $h=h^{\prime} r$. Therefore,

$$
x=e^{-h} k_{\underline{c}} e^{h}=\sum_{j=1}^{N-1}\left(c_{j} e^{-h} \lambda_{j} e^{h}-\text { H.c. }\right) .
$$

Using the notation $\operatorname{ad}_{X}(Y)=[X, Y]$ for any pair of matrices $X, Y \in \mathfrak{g} \mathfrak{t}(N)$, we first notice the identity

$$
e^{t X} Y e^{-t X}=\sum_{n=0}^{\infty} \frac{1}{n !} t^{n} a d_{X}^{n}(Y)
$$

where by $a d_{X}^{n}$ we mean the iterated application of $a d_{X}$. Indeed,

$$
\frac{d}{d t}\left(e^{t X} Y e^{-t X}\right)=e^{t X} a d_{X}(Y) e^{-t X} .
$$

Hence,

$$
\begin{aligned}
\left.\frac{d^{n}}{d t^{n}}\right|_{t=0}\left(e^{t X} Y e^{-t X}\right) & =\left.e^{t X} a d_{X}^{n}(Y) e^{-t X}\right|_{t=0} \\
& =a d_{X}^{n}(Y),
\end{aligned}
$$

so Eq. (B15) is the Taylor expansion of $e^{t X} Y e^{-t X}$. For $Y=k_{\underline{c}}, X=h$ and $t=-1$, and using that $a d_{h}\left(\lambda_{j}\right)=$ $i \alpha_{j}(h)=i \varepsilon_{j} a t$, we then have

$$
\begin{aligned}
e^{-h} \lambda_{j} e^{h} & =\sum_{n=0}^{\infty} \frac{1}{n !}(-1)^{n} a d_{h}^{n}\left(\lambda_{j}\right) \\
& =\sum_{n=0}^{\infty} \frac{1}{n !}\left(-i \varepsilon_{j} a r\right)^{n} \lambda_{j} \\
& =e^{-i \varepsilon_{j} a r} \lambda_{j} .
\end{aligned}
$$

So

$$
x=\sum_{j=1}^{N-1}\left(c_{j} e^{-i \varepsilon_{j} a r} \lambda_{j}-c_{j}^{*} e^{i \varepsilon_{j} a r} \lambda_{j}^{\dagger}\right)
$$

and

$$
\left[x, k_{\underline{c}}\right]=\sum_{j, k}\left(c_{j} c_{k} e^{-i \varepsilon_{j} a r}\left[\lambda_{j}, \lambda_{k}\right]+c_{j}^{*} c_{k}^{*} e^{i \varepsilon_{j} a r}\left[\lambda_{j}^{\dagger}, \lambda_{k}^{\dagger}\right]-c_{j} c_{k}^{*} e^{-i \varepsilon_{j} a r}\left[\lambda_{j}, \lambda_{k}^{\dagger}\right]-c_{j}^{*} c_{k} e^{i \varepsilon_{j} a r}\left[\lambda_{j}^{\dagger}, \lambda_{k}\right]\right) .
$$

By using Eq. (B4), we see that the last two terms sum up to

$$
\begin{aligned}
& -\sum_{j=1}^{N-1}\left|c_{j}\right|^{2} J_{j} i\left(e^{-i \varepsilon_{j} a r}-e^{i \varepsilon_{j} a r}\right) \\
& =-2 \sum_{j=1}^{N-1}\left|c_{j}\right|^{2} \sin \left(\varepsilon_{j} a r\right) J_{j} .
\end{aligned}
$$

On the other hand,

$$
\begin{aligned}
{\left[\lambda_{j}, \lambda_{k}\right] } & =\left[E_{j, j+1}, E_{k, k+1}\right] \\
& =\delta_{k, j+1} E_{j, j+2}-\delta_{k+1, j} E_{j, j+2},
\end{aligned}
$$

so that

$$
\begin{aligned}
\sum_{j, k} c_{j} c_{k} e^{-i \varepsilon_{j} a r}\left[\lambda_{j}, \lambda_{k}\right] \\
=\sum_{j=1}^{N-2} c_{j} c_{j+1}\left(e^{-i \varepsilon_{j} a r}-e^{-i \varepsilon_{j+1} a r}\right) E_{j, j+2},
\end{aligned}
$$

and, similarly, by taking the Hermitian conjugate,

$$
\begin{aligned}
& \sum_{j, k} c_{j}^{*} c_{k}^{*} e^{i \varepsilon_{j} a r}\left[\lambda_{j}^{\dagger}, \lambda_{k}^{\dagger}\right] \\
& =-\sum_{j=1}^{N-2} c_{j}^{*} c_{j+1}^{*}\left(e^{i \varepsilon_{j} a r}-e^{i \varepsilon_{j+1} a r}\right) E_{j+2, j} .
\end{aligned}
$$


This leads to

$$
\begin{aligned}
{\left[x, k_{\underline{c}}\right]=} & \sum_{j=1}^{N-2}\left[c_{j} c_{j+1}\left(e^{-i \varepsilon_{j} a r}-e^{-i \varepsilon_{j+1} a r}\right) E_{j, j+2}-c_{j}^{*} c_{j+1}^{*}\left(e^{i \varepsilon_{j} a r}-e^{i \varepsilon_{j+1} a r}\right) E_{j+2, j}\right] \\
& -2 \sum_{j=1}^{N-1}\left|c_{j}\right|^{2} \sin \left(\varepsilon_{j} a r\right) J_{j} .
\end{aligned}
$$

If the only nonvanishing traces are

$$
\operatorname{Tr}\left(E_{j, j+2} E_{k+2, k}\right)=\operatorname{Tr}\left(E_{j+2, j} E_{k, k+2}\right)=\delta_{j k}, \quad \operatorname{Tr}\left(J_{j} J_{k}\right)=-2 \delta_{j k}+\delta_{j, k+1}+\delta_{j+1, k},
$$

we get

$$
\begin{aligned}
\operatorname{Tr}\left(\left[x, k_{\underline{c}}\right]\left[x, k_{\underline{c}}\right]\right)= & -2 \sum_{j=1}^{N-2}\left|c_{j}\right|^{2}\left|c_{j+1}\right|^{2}\left|e^{-i \varepsilon_{j} a r}-e^{-i \varepsilon_{j+1} a r}\right|^{2}-8 \sum_{j=1}^{N-1}\left|c_{j}\right|^{4} \sin ^{2}\left(\varepsilon_{j} a r\right) \\
& +\sum_{j=1}^{N-2}\left|c_{j}\right|^{2}\left|c_{j+1}\right|^{2} \sin \left(\varepsilon_{j} a r\right) \sin \left(\varepsilon_{j+1} a r\right) .
\end{aligned}
$$

Now,

$$
\begin{aligned}
\left|e^{-i \varepsilon_{j} a r}-e^{-i \varepsilon_{j+1} a r}\right|^{2} & =2\left(1-\cos \left(\operatorname{ar}\left(\varepsilon_{j}-\varepsilon_{j+1}\right)\right)\right) \\
& =4 \sin ^{2}\left(\operatorname{ar} \frac{\varepsilon_{j}-\varepsilon_{j+1}}{2}\right) .
\end{aligned}
$$

Since $\left(\varepsilon_{j}-\varepsilon_{j+1}\right) / 2=0, \pm 1$, we can write

$$
\begin{aligned}
\sin ^{2}\left(\operatorname{ar} \frac{\varepsilon_{j}-\varepsilon_{j+1}}{2}\right) & =\sin ^{2}(\operatorname{ar})\left(\frac{\varepsilon_{j}-\varepsilon_{j+1}}{2}\right)^{2} \\
& =\frac{1}{2}\left(1-\varepsilon_{j} \varepsilon_{j+1}\right) \sin ^{2}(a r)
\end{aligned}
$$

Also

$$
\sin \left(\varepsilon_{j} a r\right) \sin \left(\varepsilon_{j+1} a r\right)=\sin ^{2}(a r) \varepsilon_{j} \varepsilon_{j+1}
$$

and $\sin ^{2}\left(\varepsilon_{j} a r\right)=\sin ^{2}(a r)$; thus, summing everything up we get Eq. (B8).

\section{APPENDIX C: $S U(2)$ VERSUS $S O(3)$}

Despite these being very well-known facts, in this appendix we discuss the difference between $S U(2)$ and $S O(3)$ since it is crucial to identify our solutions. Locally, the two groups coincide; they have the same Lie algebra. However, $S U(2)$ is simply connected, while $S O(3)$ is not. Indeed, $S U(2)$ is the universal covering of $S O(3)$. It has a nontrivial center $Z_{S U(2)}= \pm I$, with $I$ being the unit element, and there is a surjective projection

$$
\pi: S U(2) \rightarrow S O(3)
$$

with $Z_{S U(2)}$ as a kernel. Note that $S O(3)$ has a trivial kernel, and $\pi_{1}(S O(3)) \simeq Z_{S U(2)}$. We can also write

$$
S O(3) \simeq S U(2) / Z_{S U(2)}
$$

Now, let us illustrate the crucial difference we are interested in. Let $\tau_{i}, i=1,2,3$ be a canonical basis of $\operatorname{Lie}(G)$, where $G$ is one of the two groups. We can then realize the group by means of the Euler parametrization. This means that the generic element $g$ of the group has the form

$$
g(a, b, c)=e^{a \tau_{3}} e^{b \tau_{2}} e^{c \tau_{3}},
$$

where $a, b, c$ are the Euler angles. Each of the exponentials has a period (depending on the normalization of the matrices), say, $T_{3}$ for $a$ and $c$, and $T_{2}$ for $b$. The strategy to correctly cover $G$ exactly one time is explained in Ref. [40] and works as follows. To be sure to cover $G$ one integer number of times, one first allows the coordinates to run each one in the respective period. This number, in general, is larger than 1 because of redundancies, due to two reasons. The first reason is that the central element, parametrized by $b$, is chosen in the maximal torus (the exponential of the Cartan matrix). The redundancies correspond to the action of the Weyl group to the torus. This action is determined by the algebra and is the same for both $S U(2)$ and $S O(3)$. It shows that indeed moving $b$ along a period quadruplicates the determination of the points for $S U(2)$ and duplicates that for $S O(3)$, and one can reduce the range of $b$ down to $T_{2} / 4$ or $T_{2} / 2$, respectively. At this point, the difference between $S U(2)$ and $S O(3)$ appears. Indeed, for $S O(3)$ this is the end, as it is already 
covered just one time, while for $S U(2)$ it remains a redundancy and we cover it twice. This redundancy is due to the fact that

$$
e^{b \tau_{2}} \cap e^{c \tau_{3}}= \begin{cases}I & \text { if } G=S O(3) \\ \Delta=e^{\left(T_{3} / 2\right) \tau_{3}} & \text { if } G=S U(2) .\end{cases}
$$

Therefore, since $\Delta^{2}=I$,

$$
\begin{aligned}
g(a, b, c) & =e^{a \tau_{3}} e^{b \tau_{2}} e^{c \tau_{3}} \\
& =e^{a \tau_{3}} e^{b \tau_{2}} \Delta^{-2} e^{c \tau_{3}} \\
& =e^{a \tau_{3}} \Delta^{-} 1 e^{b \tau_{2}} \Delta^{-1} e^{c \tau_{3}} \\
& =g\left(a-T_{3} / 2, b, c-T_{3} / 2\right) .
\end{aligned}
$$

This redundancy is eliminated by reducing the range of $a$ down to $T_{3} / 2$ for $S U(2)$. This is the way, relevant to our case, to distinguish the two kinds of solutions: If the above intersection is $\Delta$, then the ranges of the variables $a, b, c$ are $T_{3} / 2, T_{2} / 4, T_{3}$, respectively, and the group is $S U(2)$; otherwise the ranges are $T_{3}, T_{2} / 2, T_{3}$, and the group is $S O(3)$.

Finally, we add a final remark relevant for recognizing genuine solutions: For the $S O(3)$ generator $\tau$ the orbit $\exp (x \tau)$ never meets the center, while if $\tau$ is an $S U(2)$ generator, then $\exp (x / 2 \tau)$ is the only nontrivial generator of the center of $S U(2)$. No other elements of the center of $S U(N)$ can meet these kinds of orbits.

\section{APPENDIX D: REPRESENTATIONS OF $S U(2)$ AND PERIODICITY}

It is well known from representation theory that the spin $J$ representation of $S U(2)$ has generators $T_{1}, T_{2}, T_{3}$ given by the $N \times N$ matrices, with $N=2 J+1$,

$$
\begin{aligned}
\left(T_{1}\right)_{m, n}= & \frac{i}{2} \sqrt{m(N-m)} \delta_{m, n-1} \\
& +\frac{i}{2} \sqrt{m(N-m)} \delta_{m-1, n}, \\
\left(T_{2}\right)_{m, n}= & \frac{1}{2} \sqrt{m(N-m)} \delta_{m, n-1} \\
& -\frac{1}{2} \sqrt{m(N-m)} \delta_{m-1, n}, \\
\left(T_{3}\right)_{m, n} & =i(J+1-m) \delta_{m, n} .
\end{aligned}
$$

Each of these matrices is diagonalizable, with eigenvalues given by the ones of $T_{3}$. Since

$$
U^{\dagger} \exp \left(x T_{j}\right) U=\exp \left(x U^{\dagger} T_{j} U\right)
$$

it follows that the periodicity of

$$
f_{j}(x)=\exp \left(x T_{j}\right)
$$

depends only on the eigenvalues, so all $f_{j}$ have the same periodicity, which is obviously $2 \pi$ for odd $N$ and $4 \pi$ for even $N$.

On the other hand, let us consider the matrices $k_{c}$ and $g(x)=\exp \left(x k_{\underline{c}}\right)$. The possible periodicity of $g$ depends on the eigenvalues of $k_{\underline{c}}$. It is easy to see that the coefficients of the characteristic polynomial of $k_{\underline{c}}$ depend only on the $\left|c_{j}\right|^{2}$, so the phases of $c_{j}$ are irrelevant for the periodicity. In particular, this means that the matrix $\exp \left(x \tilde{T}_{2}\right)$ with

$$
\begin{aligned}
\left(T_{2}\right)_{m, n}= & \frac{\zeta_{m}}{2} \sqrt{m(N-m)} \delta_{m, n-1} \\
& -\frac{\zeta_{n}^{*}}{2} \sqrt{n(N-n)} \delta_{m-1, n},\left|\zeta_{j}\right|=1,
\end{aligned}
$$

has the same periodicity of $f_{2}(x)$.

\section{APPENDIX E: SOLVING THE PERIODICITY PROBLEM}

In Sec. IV we showed that for $N$ higher than 3 , there is a further difficulty to overcome in order to find a global solution: Generically, the matrix $g(x)=e^{x k}$ is not periodic, and its orbit densely fills a torus of dimension strictly higher than 1 . This phenomenon corresponds to the fact that the one parameter subgroup $g(x)$ is not a Lie subgroup but only an imbedded subgroup. Therefore, for arbitrary choices of the coefficients $c_{j}$, the matrix

$$
k_{\underline{c}}=\sum_{j=1}^{N-1}\left(c_{j} E_{j, j+1}-c_{j}^{*} E_{j+1, j}\right)
$$

cannot be used to generate global solutions unless the corresponding $g(x)$ is periodic. We now tackle this problem, in general. For the sake of completeness, we first show that no problems arise in the case $N=3$.

\section{The case $N=3$}

In this simple case we have

$$
k_{\underline{c}}=\left(\begin{array}{ccc}
0 & c_{1} & 0 \\
-c_{1}^{*} & 0 & c_{2} \\
0 & -c_{2}^{*} & 0
\end{array}\right) .
$$

The corresponding characteristic polynomial is

$$
P_{k}(\lambda):=\operatorname{det}\left(\lambda \mathbb{I}-k_{\underline{c}}\right)=\lambda\left(\lambda^{2}+\|\underline{c}\|^{2}\right) .
$$

The eigenvalues are therefore $0, \pm i\|\underline{c}\|$, which are in rational ratios so $g(\gamma)=\exp \left(\gamma k_{c}\right)$ is periodic, in particular, with period $2 \pi /\|\underline{c}\|$. For other purposes, we explicitly 
compute $g(\gamma)$. To this aim, let us first notice that, using the Cayley-Hamilton theorem, $k_{\underline{c}}$ satisfies

$$
k_{\underline{c}}\left(k_{\underline{c}}^{2}+\|\underline{c}\|^{2} \mathbb{I}\right)=\mathbb{O},
$$

where $\mathbb{I}$ and $\mathbb{O}$ are the identity and the null $3 \times 3$ matrices. This implies $k_{\underline{c}}^{3}=-\|\underline{c}\|^{2} k_{\underline{c}}$ so that any power of $k_{\underline{c}}$ can be reduced to a power lower than 3 . Hence,

$$
e^{\gamma k_{\underline{c}}}=g_{1}(\gamma) \mathbb{I}+g_{2}(\gamma) k_{\underline{c}}+g_{3}(\gamma) k_{\underline{c}}^{2},
$$

for three functions satisfying $g_{1}(0)=1, g_{2}(0)=g_{3}(0)=0$, since $e^{\mathbb{O}}=\mathbb{I}$. Deriving Eq. (E5) with respect to $\gamma$ and using the characteristic equation, we get

$$
\begin{aligned}
& g_{1}^{\prime}(\gamma) \mathbb{I}+g_{2}^{\prime}(\gamma) k_{\underline{c}}+g_{3}^{\prime}(\gamma) k_{\underline{c}}^{2}=k_{\underline{c}} e^{\gamma k_{\underline{c}}} \\
& \quad=g_{1}(\gamma) k_{\underline{c}}+g_{2}(\gamma) k_{\underline{c}}^{2}+g_{3}(\gamma) k_{\underline{c}}^{3} \\
& \quad=\left(g_{1}(\gamma)-\|\underline{c}\|^{2} g_{3}(\gamma)\right) k_{\underline{c}}+g_{2}(\gamma) k_{\underline{c}}^{2},
\end{aligned}
$$

so that

$$
\begin{gathered}
g_{1}^{\prime}(\gamma)=0, \\
g_{2}^{\prime}(\gamma)=g_{1}(\gamma)-\|\underline{c}\|^{2} g_{3}(\gamma), \\
g_{3}^{\prime}(\gamma)=g_{2}(\gamma),
\end{gathered}
$$

with the Cauchy conditions $g_{1}(0)=1, g_{2}(0)=g_{3}(0)=0$ [so that $g_{2}^{\prime}(0)=1$ ]. From the first equation we immediately get $g_{1}(\gamma)=1$, while deriving the second one and replacing from the third, we get

$g_{2}^{\prime \prime}(\gamma)=-\|\underline{c}\|^{2} g_{2}(\gamma), \quad g_{2}(0)=0, \quad g_{2}^{\prime}(0)=1$,

with the solution

$$
g_{2}(\gamma)=\frac{\sin (\|\underline{c}\| \gamma)}{\|\underline{c}\|}
$$

Finally, from the third equation we get

$$
\begin{aligned}
g_{3}(\gamma) & =\int_{0}^{\gamma} d x \frac{\sin (\|\underline{c}\| \gamma)}{\|\underline{c}\|}=\frac{1-\cos (\|\underline{c}\| \gamma)}{\|\underline{c}\|^{2}} \\
& =2 \frac{\sin ^{2}\left(\frac{\|\underline{c}\|}{2} \gamma\right)}{\|\underline{c}\|^{2}} .
\end{aligned}
$$

Therefore,

$$
e^{\gamma k_{\underline{c}}}=I+\frac{\sin (\|\underline{c}\| \gamma)}{\|\underline{c}\|} k_{\underline{c}}+2 \frac{\sin ^{2}\left(\frac{\|\underline{c}\|}{2} \gamma\right)}{\|\underline{c}\|^{2}} k_{\underline{c}}^{2} .
$$

\section{The general case}

One can, in principle, solve this problem as follows. Since $k$ is anti-Hermitian, it can be diagonalized in $\mathbb{C}$, with pure imaginary eigenvalues. Moreover, if $\lambda$ is an eigenvalue, so is $-\lambda=\lambda^{*}$. Therefore, if $S$ is the integer part of $N / 2$ (so that $N=2 S$ or $N=2 S+1$ for $N$ even and odd, respectively), generically we have $S$ distinct nonvanishing eigenvalues. Let $U$ be a unitary matrix such that

$$
k=U^{\dagger} \sigma U,
$$

where $\sigma$ is the diagonal form of $k$, say,

$$
\sigma= \begin{cases}\operatorname{diag}\left(i \lambda_{1},-i \lambda_{1}, \ldots, i \lambda_{S},-i \lambda_{S}\right) & \text { N even } \\ \operatorname{diag}\left(i \lambda_{1},-i \lambda_{1}, \ldots, i \lambda_{S},-i \lambda_{S}, 0\right) & \text { N odd }\end{cases}
$$

with $\lambda_{j}>0$. Since

$$
e^{x k}=e^{x U^{\dagger} \sigma U}=U^{\dagger} e^{x \sigma} U,
$$

$e^{x k}$ is periodic if and only if $e^{x \sigma}$ is. Now, $e^{T \sigma}$ is the identity if and only if

$$
e^{i T \lambda_{j}}=1
$$

for all $j=1, \ldots, S$; that means $T \lambda_{j}=n_{j} 2 \pi$, with $n_{j}$ a positive integer (obviously, we assume $T>0$ ) for any $j=1, \ldots, S$. Therefore,

$$
\frac{\lambda_{j}}{\lambda_{k}}=\frac{n_{j}}{n_{k}},
$$

so all pairs of eigenvalues must have rational quotients. Of course, this condition is satisfied for $N \leq 3$, and any choice of $\underline{c}$ is allowed. But for $N \geq 4$ we cannot choose the $c_{j}$ arbitrarily: Only those values such that $k$ admits eigenvalues with rational ratios are allowed. Notice that $\underline{c}$ remains defined up to a real multiplicative constant: If $t \in \mathbb{R}$, then $k_{t \underline{c}}=t k_{c}$.

The eigenvalues are the solutions of the characteristic polynomial

$$
P_{N}(x)=\operatorname{det}\left(x \mathbb{I}-k_{\underline{c}}\right),
$$

of degree $N$ in $x$. Since $k_{c}$ is anti-Hermitian, its eigenvalues are purely imaginary and, moreover, if $\mu$ is a nonvanishing eigenvalue, then $\mu^{*}=-\mu$ is also an eigenvalue. So the nonvanishing eigenvalues are in pairs and, if $N$ is odd, there is at least one zero eigenvalue. Moreover, since in the factorization of the polynomial the nonvanishing eigenvalues $\mu$ must appear in the factors $(x-\mu)(x+\mu)=x^{2}-\mu^{2}$, we see that the general form of the polynomial must be 


$$
P_{N}(x)= \begin{cases}\left(x^{2}\right)^{n}+a_{1}\left(x^{2}\right)^{n-1}+\cdots+a_{n} & \text { for } N=2 n \\ x\left[\left(x^{2}\right)^{n}+a_{1}\left(x^{2}\right)^{n-1}+\cdots+a_{n}\right] & \text { for } N=2 n+1 .\end{cases}
$$

The coefficients $a_{j}$ are not the same for $N$ odd and for $N$ even, but it is convenient to keep the same name so that we can generically write the equation for the nonvanishing eigenvalues as

$y^{n}+a_{1} y^{n-1}+\cdots+a_{n-1} y+a_{n}=0, \quad y=x^{2}$.

We can be more precise with the following proposition.

Proposition 6: Using the notation $j \ll k$ for $k-j \geq 2$, we have

$$
\begin{gathered}
a_{1}=\|\underline{c}\|^{2}, \\
a_{k}=\sum_{j_{1} \ll j_{2} \ll \ldots \ll j_{k}}\left|c_{j_{1}}\right|^{2}\left|c_{j_{2}}\right|^{2} \cdots\left|c_{j_{k}}\right|^{2}, \quad k=2, \ldots, n .
\end{gathered}
$$

Proof.-It can be easily proven by induction. We have already seen this for $N=3$. A direct computation shows that it is true also for $N=4$ since $P_{4}(x)=x^{4}+$ $x^{2}\left(\left|c_{1}\right|^{2}+\left|c_{2}\right|^{2}+\left|c_{3}\right|^{2}\right)+\left|c_{1}\right|^{2}\left|c_{3}\right|^{2}$. Now, assume this is true for $N$ and $N-1$. Let $k_{n}$ be the matrix $n \times n$ defined as $k_{\underline{c}}$ with components $c_{1}, \ldots, c_{n}$. This way, we see $k_{n}$ as a submatrix of $k_{n+1}$ obtained by erasing the last row and column. Let

$$
P_{n}(x)=\operatorname{det}\left(x \mathbb{I}_{n \times n}-k_{n}\right) .
$$

Developing the determinant with the Laplace rule applied to the last row, we easily find

$$
P_{N+1}(x)=x P_{N}(x)+\left|c_{N}\right|^{2} P_{N-1}(x) \text {. }
$$

The first addendum contains all the monomials of the stated form except the terms containing $\left|c_{N}\right|^{2}$. The second addendum contains all the terms of the stated form containing $\left|c_{N}\right|^{2}$. This completes our proof.

So, for example,

$$
\begin{aligned}
P_{4}(x)= & x^{4}+x^{2}\left(\left|c_{1}\right|^{2}+\left|c_{2}\right|^{2}+\left|c_{3}\right|^{2}\right)+\left|c_{1}\right|^{2}\left|c_{3}\right|^{2}, \\
P_{5}(x)= & x\left(x^{4}+x^{2} \|\left.\underline{c}\right|^{2}\right. \\
& \left.+\left(\left|c_{4}\right|^{2}\left|c_{1}\right|^{2}+\left|c_{4}\right|^{2}\left|c_{2}\right|^{2}+\left|c_{3}\right|^{2}\left|c_{1}\right|^{2}\right)\right),
\end{aligned}
$$

$$
P_{6}(x)=x^{6}+\|\underline{c}\|^{2} x^{4}+x^{2}\left(\left|c_{4}\right|^{2}\left(\left|c_{1}\right|^{2}+\left|c_{2}\right|^{2}\right)+\left|c_{1}\right|^{2}\left|c_{3}\right|^{2}+\left|c_{5}\right|^{2}\left(\left|c_{1}\right|^{2}+\left|c_{2}\right|^{2}+\left|c_{3}\right|^{2}\right)\right)+\left|c_{1} c_{3} c_{5}\right|^{2} \text {. }
$$

$$
a_{2}=\sum_{j_{1}<j_{2}}\left(-y_{j_{1}}\right)\left(-y_{j_{2}}\right)
$$
Now, condition (E18) is equivalent to requiring that there must exist a positive real number $z$ and $n$ positive integers $m_{j}, j=1, \ldots, n$ such that the nonvanishing eigenvalues of $k_{\underline{c}}$ must have the form $\lambda_{j}^{ \pm}= \pm i m_{j} z$. This happens if the solutions of Eq. (E21) are

$$
y_{j}=-z^{2} m_{j}^{2}
$$

At this point, we notice that the coefficient of the above polynomial can be written in terms of the solutions as

$$
a_{1}=-\sum_{j=1}^{N} y_{j}
$$

Comparing with the last proposition, we get the following set of equations for $\left|c_{j}^{2}\right|=: \zeta_{j}$ : 


$$
\begin{gathered}
\sum_{j=1}^{N-1} \zeta_{j}=z^{2} \sum_{a=1}^{n} m_{a}^{2}, \\
\sum_{j_{1} \ll \ldots j_{k} \leq N-1} \zeta_{j_{1}} \cdots \zeta_{j_{k}}=z^{2 k} \sum_{a_{1}<\ldots<a_{k} \leq n} m_{a_{1}}^{2} \cdots m_{a_{k}}^{2}, \quad k=2, \ldots, n .
\end{gathered}
$$

This is a set of $n$ equations in $N-1$ real positive variables. We now show that it generically has an $(N-1-n)$ dimensional space of solutions in the interesting region, which is positive for $\zeta_{j}$. To this end, we assume the generic situation where all $m_{a}$ are different, and we order them in an increasing sequence $m_{1}<m_{2}<\cdots<m_{n}$. We will show later that the condition on the $m_{a}$ cannot be weakened in order to get periodic solutions. Then, we show that there is a simple solution on the boundary of the region of interest, which is (if $N$ is odd, we assume the null eigenvalue to be the last one, $\lambda_{2 n+1}=0$ )

$$
\zeta_{2 a}=0, \quad \zeta_{2 a-1}=z^{2} m_{a}^{2}, \quad a=1, \ldots, n .
$$

Next, we claim that starting from this point, we can find a smooth family of solutions $\zeta_{2 a-1}\left(\left\{\zeta_{2 b}\right\}\right)$ in a small open neighborhood of $\zeta_{2 b}=0$. In particular, it implies that there are positive (by continuity) $\zeta_{2 a-1}$ 's parametrized by small positive $\zeta_{b}$ 's. This is sufficient to show that there is generically a moduli space of real dimension $N-n-1$ for the solutions for the above system.

Proof of the claim.-To prove the claim, let us consider the functions

$$
\begin{gathered}
F_{1}\left(\zeta_{1}, \ldots, \zeta_{N-1}\right)=\sum_{j=1}^{N-1} \zeta_{j}, \\
F_{k}\left(\zeta_{1}, \ldots, \zeta_{N-1}\right)=\sum_{j_{1} \ll \ldots \ll j_{k} \leq N-1} \zeta_{j_{1}} \cdots \zeta_{j_{k}}, \\
k=2, \ldots, n,
\end{gathered}
$$

and the square submatrix $M$ of its Jacobian defined by

$$
M_{a, b}=\left.\frac{\partial F_{a}}{\partial \zeta_{2 b-1}}\right|_{\zeta_{j}=\bar{z}_{j}},
$$

where $\bar{\zeta}_{j}$ are defined by Eq. (E36). Therefore, we have

$$
\begin{gathered}
M_{1, b}=1, \\
M_{2, b}=\sum_{c \neq b} z^{2} m_{c}^{2}, \\
M_{3, b}=\sum_{\substack{c_{1}<c_{2} \\
c_{j} \neq b,}} z^{4} m_{c_{1}}^{2} m_{c_{2}}^{2},
\end{gathered}
$$

and, more in general,

$$
\begin{aligned}
M_{k, b}-M_{k, 1}= & \sum_{\substack{c_{1}<\ldots<c_{k-1}, c_{j} \neq b,-}} z^{2 k-2} m_{c_{1}}^{2} \cdots m_{c_{k-1}}^{2}, \\
& -\sum_{\substack{c_{1}<\ldots<c_{k-1}, c_{j} \neq 1,}} z^{2 k-2} m_{c_{1}}^{2} \cdots m_{c_{k-1}}^{2}, \\
= & z^{2}\left(m_{b}^{2}-m_{1}^{2}\right) \sum_{\substack{c_{1} \ldots<c_{k-2}, 1 \neq c_{j} \neq b .}} m_{c_{1}}^{2} \cdots m_{c_{k-2}}^{2},
\end{aligned}
$$

We want to compute the determinant of this matrix. It does not change if we subtract the first column from all the other ones. In doing this, the first line becomes $\delta_{1, j}$, so we can compute the determinant by applying the Laplace formula to the first line. So, the determinant is equal to the determinant of the new matrix with the first row and the first column canceled out. To understand how this matrix appears, let us note that the second row is

$M_{2, b}-M_{2,1}=\sum_{c \neq b} z^{2} m_{c}^{2}-\sum_{c \neq 1} z^{2} m_{c}^{2}=z^{2}\left(m_{b}^{2}-m_{1}^{2}\right)$,

Therefore, from the $b$ th column of the reduced matrix, $b=$ $2, \ldots, n$ has a factor $z^{2}\left(m_{b}^{2}-m_{1}^{2}\right)$, and since the determinant is multilinear on the columns, we get

$$
\operatorname{det}(M)=\prod_{b=2}^{n} z^{2}\left(m_{b}^{2}-m_{1}^{2}\right) \operatorname{det}(\tilde{M})
$$

where $\tilde{M}$ is an $(n-1) \times(n-1)$ matrix whose first row has all elements equal to 1 and 


$$
\tilde{M}_{k, b}=\sum_{\substack{c_{1}<<<c_{k-1}, . \\ 1 \neq c_{j} \neq b_{1}}} z^{2 k-2} m_{c_{1}}^{2} \cdots m_{c_{k-1}}^{2}
$$

In other words, we see that $\tilde{M}$ is like $M$ but in one lower dimension and where $m_{1}$ has disappeared. We can then inductively repeat the same construction, finally arriving at the conclusion

$$
\operatorname{det}(M)=\prod_{a<b} z^{2}\left(m_{b}^{2}-m_{a}^{2}\right) .
$$

Since $m_{a}^{2}<m_{b}^{2}$ for $a<b$, we see that this determinant is different from zero. The proof of the claim then is an immediate consequence of the implicit function theorem.

Going back to $c_{j}$, we then see that, in general,

$$
c_{j}=\xi_{j} \sqrt{\zeta_{j}(\underline{m}, \underline{t})},
$$

for arbitrary phases $\xi_{j}, j=1, \ldots, N-1$, with $\underline{m} \in \mathbb{N}_{>}^{n}$, $\underline{t} \in W \subset \mathbb{R}^{N-n-1}$. The parameters $t_{j}$ parametrize the above family of solutions. We can always assume that the integer $m_{j}$ is coprime. Indeed, if $m$ is a common divisor of $m_{j}$ so that $m_{j}=m s_{j}$, then we can write $\underline{m}=m \underline{s}$ and $m$ can be reabsorbed in $z$. Having assumed this, we can now fix $z$ in such a way that $e^{x k_{\underline{c}}}$ has period $2 \pi$. Indeed, since the nonvanishing eigenvalues of $k_{\underline{c}}$ are $\lambda_{j}^{ \pm}= \pm i z m_{j}$, since the $m_{j}$ is coprime, the common period of the associated exponential is $2 \pi / z$. This fixes $z=1$.

Notice, in particular, that in this case

$$
\|\underline{c}\|^{2}=\sum_{j=1}^{n} m_{j}^{2} \equiv\|\underline{m}\|^{2} .
$$

The associated baryon number is

$$
B=2 \sigma m\|\underline{m}\|^{2} .
$$

We have proved the following proposition.

Proposition 7. For $N=2 n$ or $N=2 n+1$ and for any $n$-tuple of strictly increasing coprime positive integers $m_{a}$, $a=1, \ldots, n$, the matrices $k_{\underline{c}}$, such that $e^{x k_{c}}$ has period $2 \pi$, are a family of dimension $2 N-2+n$, where $n$ is the integer part of $N / 2$. Beyond $\underline{m}$, this family is described by $N-1$ phases and by $N-n-1$ real parameters varying in a set $W$, parametrizing the solutions of the system,

$$
\begin{gathered}
\sum_{j=1}^{N-1} \zeta_{j}=\sum_{a=1}^{n} m_{a}^{2}, \\
\sum_{j_{1} \ll \ldots \ll j_{k} \leq N-1} \zeta_{j_{1}} \cdots \zeta_{j_{k}}=\sum_{\substack{a_{1}<\ldots<a_{k} \leq n \\
k=2, \ldots, n .}} m_{a_{1}}^{2} \cdots m_{a_{k}}^{2},
\end{gathered}
$$

Correspondingly, the fundamental baryon number is $B_{0}=$ $2 \sigma\|\underline{m}\|^{2}$.

One says that these matrices have a moduli space

$$
\mathcal{M}=\mathbb{T}^{N-1} \times W,
$$

where $\mathbb{T}^{N-1}$ is the torus generated by the phases and $W \subset$ $\mathbb{R}^{N-n-1}$ is the moduli space of the system. It is difficult to say something general about the global properties of $W$. We study, in general, the case $N=4$ where all computations are explicitly feasible.

Remark: For $N=3$ we have $n=1$ and, therefore, only one integer $m$ must be equal to 1 ("coprime"). So $\underline{c}$ must have norm 1 , and the fundamental baryon number is $B=2 \sigma$.

\section{The $N=4$ case}

Let us apply the above results to the case of $S U(4)$. We have $n=2$, so we expect the dimension of $W$ to be 1 . The eigenvalue equation for $k$ is

$$
0=\lambda^{4}+\lambda^{2}\|\underline{c}\|^{2}+\left|c_{1}\right|^{2}\left|c_{3}\right|^{2} .
$$

The four solutions are $i \lambda_{+}, i \lambda_{-},-i \lambda_{+},-i \lambda_{-}$, with

$$
\lambda_{ \pm}=\sqrt{\frac{\|\underline{\underline{c}}\|^{2}}{4}+\frac{\left|c_{1}\right|\left|c_{3}\right|}{2}} \pm \sqrt{\frac{\|\underline{c}\|^{2}}{4}-\frac{\left|c_{1}\right|\left|c_{3}\right|}{2}} .
$$

Let $q \leq p$ be a pair of positive coprime integer numbers. Then, we have to solve the system

$$
\begin{gathered}
\zeta_{1}+\zeta_{2}+\zeta_{3}=p^{2}+q^{2}, \\
\zeta_{1} \zeta_{3}=p^{2} q^{2} .
\end{gathered}
$$

Notice that this gives

$$
\lambda_{+}=p, \quad \lambda_{-}=q .
$$

Now, let us replace

$$
\zeta_{3}=p^{2} q^{2} / \zeta_{1}
$$

in the first equation, so that

$$
\zeta_{1}+\frac{p^{2} q^{2}}{\zeta_{1}}-\left(p^{2}+q^{2}\right)=-\zeta_{2}
$$

Since we have to require $\zeta_{2}>0$, we see that it must be

$$
\zeta_{1}^{2}-\left(p^{2}+q^{2}\right) \zeta_{1}+p^{2} q^{2}<0 .
$$

This is equivalent to saying

$$
q^{2}<\zeta_{1}<p^{2} .
$$


So we can use $\tau=\sqrt{\zeta_{1}}$ as a modulus to represent $W$. The moduli space, including the boundary, is therefore

$$
\mathcal{M}_{4}=\mathbb{T}^{3} \times[q, p] .
$$

For

$$
\left(e^{i \alpha_{1}}, e^{i \alpha_{2}}, e^{i \alpha_{3}}, \tau\right) \in \mathcal{M}_{4},
$$

we have

$\underline{c}=\left(e^{i \alpha_{1}} \tau ; e^{i \alpha_{2}} \sqrt{p^{2}+q^{2}-\tau^{2}-\frac{p^{2} q^{2}}{\tau^{2}}} ; \frac{p q}{\tau} e^{i \alpha_{3}}\right)$.

The corresponding period is of course

$$
T=2 \pi,
$$

and the fundamental baryon number is

$$
B_{0}=2 \sigma\left(p^{2}+q^{2}\right) .
$$

Finally, we can compute the exponential. Rewriting the characteristic polynomial as

$$
P(x)=x^{4}+x^{2}\left(p^{2}+q^{2}\right)+p^{2} q^{2},
$$

we see that the matrix $k \equiv k_{\underline{c}}$ satisfies

$$
k^{4}=-\left(p^{2}+q^{2}\right) k^{2}-p^{2} q^{2} \mathbb{I} .
$$

This implies that there must exist four functions $f_{j}(x)$, $j=0,1,2,3$ such that

$$
e^{x k}=f_{0}(x) \mathbb{I}+f_{1}(x) k+f_{2}(x) k^{2}+f_{3}(x) k^{3},
$$

with $f_{0}(0)=1, f_{a}(0)=0, a=1,2,3$. From

$$
\frac{d}{d x} e^{x k}=k e^{x k}
$$

we get

$$
\begin{aligned}
& f_{0}^{\prime}(x) \mathbb{I}+f_{1}^{\prime}(x) k+f_{2}^{\prime}(x) k^{2}+f_{3}^{\prime}(x) k^{3} \\
& =f_{0}(x) k+f_{1}(x) k^{2}+f_{2}(x) k^{3} \\
& \quad+f_{3}(x)\left(-\left(p^{2}+q^{2}\right) k^{2}-p^{2} q^{2} \mathbb{I}\right),
\end{aligned}
$$

which gives the system of differential equations

$$
\begin{gathered}
f_{0}^{\prime}=-p^{2} q^{2} f_{3}, \\
f_{1}^{\prime}=f_{0},
\end{gathered}
$$

$$
\begin{gathered}
f_{2}^{\prime}=f_{1}-\left(p^{2}+q^{2}\right) f_{3}, \\
f_{3}^{\prime}=f_{2},
\end{gathered}
$$

with the Cauchy conditions $f_{j}(0)=\delta_{j, 0}$. Using the fourth equation in the third one, we get

$$
f_{3}^{\prime \prime}=f_{1}-\left(p^{2}+q^{2}\right) f_{3}, \quad f_{3}^{\prime \prime}(0)=0 .
$$

Deriving this again using the second equation, we get

$$
f_{3}^{\prime \prime \prime}=f_{0}-\left(p^{2}+q^{2}\right) f_{3}^{\prime}, \quad f_{3}^{\prime \prime \prime}(0)=1 .
$$

Deriving this a last time using the first equation, we finally get the Cauchy problem

$$
f_{3}^{\prime \prime \prime}+\left(p^{2}+q^{2}\right) f_{3}^{\prime \prime}+p^{2} q^{2} f_{3}=0
$$

$f_{3}(0)=0, f_{3}^{\prime}(0)=0, \quad f_{3}^{\prime \prime}(0)=0, \quad f_{3}^{\prime \prime \prime}(0)=1$.

This is easily solved, and it also gives $f_{2}=f_{3}^{\prime}$, $f_{1}=f_{2}^{\prime}+\left(p^{2}+q^{2}\right) f_{3}$, and finally $f_{0}=f_{1}^{\prime}$. For $p>q$, we get

$$
f_{0}(x)=\frac{p^{2}}{p^{2}-q^{2}} \cos (q x)-\frac{q^{2}}{p^{2}-q^{2}} \cos (p x),
$$

$f_{1}(x)=\frac{p^{2}}{q\left(p^{2}-q^{2}\right)} \sin (q x)-\frac{q^{2}}{p\left(p^{2}-q^{2}\right)} \sin (p x)$,

$$
\begin{aligned}
& f_{2}(x)=\frac{1}{p^{2}-q^{2}}(\cos (q x)-\cos (p x)), \\
& f_{3}(x)=\frac{1}{p^{2}-q^{2}}\left(\frac{\sin (q x)}{q}-\frac{\sin (p x)}{p}\right) .
\end{aligned}
$$

In the case $p=q=1$ we have

$$
f_{3}(x)=-\frac{1}{2} x \cos x+\frac{1}{2} \sin x .
$$

This is sufficient to show that the case $p=q$ must be excluded, since the solution is no longer periodic.

\section{APPENDIX F: BARYONIC NUMBER}

The baryon number is defined by the integral

$$
B=\frac{1}{24 \pi^{2}} \int \epsilon^{i j k} \operatorname{Tr}\left(R_{i} R_{j} R_{k}\right) \sqrt{g} d r d \phi d \gamma .
$$

Now, 


$$
\begin{aligned}
\epsilon^{i j k} \operatorname{Tr}\left(R_{i} R_{j} R_{k}\right) & =\frac{3}{L_{r} L_{\gamma} L_{\varphi}} \epsilon^{r \gamma \phi} \operatorname{Tr}\left(R_{r}\left[R_{\gamma}, R_{\varphi}\right]\right) \\
& =-\frac{3 \sigma m}{L_{r} L_{\gamma} L_{\varphi}} \operatorname{Tr}\left(h^{\prime}\left[k_{\underline{c}}, x\right]\right),
\end{aligned}
$$

where we used the explicit expressions for the $R_{a}$. After using Eq. (B25), we get

$$
\epsilon^{i j k} \operatorname{Tr}\left(R_{i} R_{j} R_{k}\right)=-\frac{6 \sigma m}{L_{r} L_{\gamma} L_{\varphi}} \sum_{j=1}^{N-1}\left|c_{j}\right|^{2} \varepsilon_{j} \sin (a r) \operatorname{Tr}\left(h^{\prime} J_{j}\right),
$$

and using

$$
-\varepsilon_{j} \operatorname{Tr}\left(h^{\prime} J_{j}\right)=a,
$$

we finally get

$$
\epsilon^{i j k} \operatorname{Tr}\left(R_{i} R_{j} R_{k}\right)=\frac{6 \sigma m}{\sqrt{g}}\|\underline{c}\|^{2} a \sin (a r) .
$$

Replacing this in the integral and integrating, we get

$$
B=2 m \sigma\|\underline{\underline{c}}\|^{2} .
$$

Remark: The form

$$
\omega=\epsilon^{i j k} \operatorname{Tr}\left(R_{i} R_{j} R_{k}\right) \sqrt{g} d r d \phi d \gamma
$$

is nothing but the pullback on the rectangular box of the volume form $\operatorname{Tr}(R \wedge R \wedge R)$ over the cycle; see, for example, Ref. [39].

\section{APPENDIX G: MINIMAL ENERGY PER BARYON}

Let us minimize expression (4.53) with respect to the $L_{a}$, $a=\varphi, r, \gamma$. Let us rewrite it in the form

$$
g\left(L_{\varphi}, L_{r}, L_{\gamma}\right)=D L_{\varphi} L_{r} L_{\gamma}\left[\frac{A^{2}}{L_{\varphi}^{2}}+\frac{B^{2}}{L_{r}^{2}}+\frac{C^{2}}{L_{\varphi}^{2} L_{r}^{2}}+\frac{M^{2}}{L_{\gamma}^{2}}\left(1+\frac{\alpha^{2}}{L_{\varphi}^{2}}+\frac{\beta^{2}}{L_{r}^{2}}\right)\right],
$$

where

$$
\begin{aligned}
& D=\frac{K \pi^{3}}{4 \sigma m}, \quad A=4 \sigma, \quad B=\frac{\left\|v_{\underline{\varepsilon}}\right\|}{\|\underline{c}\|}, \\
& C=\sigma \sqrt{\lambda}, \quad M=2 \sqrt{2} m, \quad \beta=\frac{\sqrt{\lambda}}{4}, \\
& \alpha=\sqrt{\lambda} \frac{\sigma}{\|\underline{c}\|}\left(\sum_{j=1}^{N-1}\left|c_{j}\right|^{4}+\sum_{j=1}^{N-2}\left|c_{j}\right|^{2}\left|c_{j+1}\right|^{2}\left(\frac{1}{2}-\frac{3}{2} \varepsilon_{j} \varepsilon_{j+1}\right)\right)^{\frac{1}{2}} .
\end{aligned}
$$

Deriving with respect to $L_{j}$ and setting

$$
x=\frac{1}{L_{\varphi}^{2}}, \quad y=\frac{1}{L_{r}^{2}}, \quad z=\frac{M^{2}}{L_{\gamma}^{2}},
$$

we get the equations for the stationary points:

$$
\begin{aligned}
& A^{2} x+B^{2} y+C^{2} x y-z\left(1+\alpha^{2} x+\beta^{2} y\right)=0, \\
& A^{2} x-B^{2} y+C^{2} x y-z\left(1-\alpha^{2} x+\beta^{2} y\right)=0, \\
& -A^{2} x+B^{2} y+C^{2} x y-z\left(1+\alpha^{2} x-\beta^{2} y\right)=0 .
\end{aligned}
$$

Solving the first equation with respect to $z$ and replacing this in the remaining equations, we get

$$
\begin{gathered}
z=\frac{A^{2} x+B^{2} y+C^{2} x y}{1+\alpha^{2} x+\beta^{2} y}, \\
0=B^{2} y+B^{2} \beta^{2} y^{2}-\alpha^{2} x^{2}\left(A^{2}+C^{2} y\right), \\
0=A^{2} x\left(1+\alpha^{2} x\right)-\beta^{2} y^{2}\left(B^{2}+C^{2} x\right) .
\end{gathered}
$$

From the third equation we get

$$
y^{2}=\frac{A^{2} x}{\beta^{2}} \frac{1+\alpha^{2} x}{B^{2}+C^{2} x}
$$

which, when replaced in the second term of the second equation, gives

$$
\left(\alpha^{2} x^{2} C^{2}-B^{2}\right)\left(y+\frac{A^{2} x}{B^{2}+C^{2} x}\right)=0 .
$$


Since we are looking for positive $x, y, z$, the second factor is strictly positive, and the only allowed solution is $x=\frac{B}{\alpha C}$. Replacing in Eq. (G10) and then in Eq. (G7), we get

$$
x=\frac{B}{\alpha C}, \quad y=\frac{A}{\beta C}, \quad z=\frac{A B}{\alpha \beta} . \quad(\mathrm{G} 12) \quad \frac{1}{L_{\gamma}^{2}}=\frac{2\left\|v_{\underline{\varepsilon}}\right\|}{\lambda m^{2}}
$$

Therefore,

$$
\times\left(\sum_{j=1}^{N-1}\left|c_{j}\right|^{4}+\sum_{j=1}^{N-2}\left|c_{j}\right|^{2}\left|c_{j+1}\right|^{2}\left(\frac{1}{2}-\frac{3}{2} \varepsilon_{j} \varepsilon_{j+1}\right)\right)^{-\frac{1}{2}},
$$

$\frac{1}{L_{\varphi}^{2}}=\frac{\left\|v_{\underline{\varepsilon}}\right\|}{\lambda \sigma^{2}}\left(\sum_{j=1}^{N-1}\left|c_{j}\right|^{4}+\sum_{j=1}^{N-2}\left|c_{j}\right|^{2}\left|c_{j+1}\right|^{2}\left(\frac{1}{2}-\frac{3}{2} \varepsilon_{j} \varepsilon_{j+1}\right)\right)^{-\frac{1}{2}}$,

(G13)

$$
\frac{1}{L_{r}^{2}}=\frac{16}{\lambda}
$$

and the corresponding energy per baryon in standard units $\left[K=\left(6 \pi^{2}\right)^{-1}, \lambda=1\right]$ is

$$
g(\underline{c}, \varepsilon)=\frac{\pi}{3 \sqrt{2}}\left[2+\frac{\left\|v_{\underline{\varepsilon}}\right\|}{\|\underline{c}\|^{2}}\left(\sum_{j=1}^{N-1}\left|c_{j}\right|^{4}+\sum_{j=1}^{N-2}\left|c_{j}\right|^{2}\left|c_{j+1}\right|^{2}\left(\frac{1}{2}-\frac{3}{2} \varepsilon_{j} \varepsilon_{j+1}\right)\right)^{\frac{1}{2}}\right] .
$$

[1] N. Brambilla et al., Eur. Phys. J. C 74, 2981 (2014).

[2] Y. Nambu and G. Jona-Lasinio, Phys. Rev. 122, 345 (1961).

[3] K. Rajagopal and F. Wilczek, in At the Frontier of Particle Physics. Handbook of QCD. Vol. 1-3, edited by M. Shifman and B. Ioffe (World Scientific, Singapore, 2000), pp. 2061-2151.

[4] M. G. Alford, J. A. Bowers, and K. Rajagopal, Phys. Rev. D 63, 074016 (2001).

[5] R. Casalbuoni and G. Nardulli, Rev. Mod. Phys. 76, 263 (2004).

[6] D. G. Ravenhall, C. J. Pethick, and J. R. Wilson, Phys. Rev. Lett. 50, 2066 (1983).

[7] M. Hashimoto, H. Seki, and M. Yamada, Prog. Theor. Phys. 71, 320 (1984).

[8] C. J. Horowitz, D. K. Berry, C. M. Briggs, M. E. Caplan, A. Cumming, and A. S. Schneider, Phys. Rev. Lett. 114, 031102 (2015).

[9] D. K. Berry, M. E. Caplan, C. J. Horowitz, G. Huber, and A. S. Schneider, Phys. Rev. C 94, 055801 (2016).

[10] C. O. Dorso, G. A. Frank, and J. A. López, Nucl. Phys. A978, 35 (2018).

[11] A. da Silva Schneider, M. E. Caplan, D. K. Berry, and C. J. Horowitz, Phys. Rev. C 98, 055801 (2018).

[12] M. E. Caplan, A. S. Schneider, and C. J. Horowitz, Phys. Rev. Lett. 121, 132701 (2018).

[13] R. Nandi and S. Schramm, J. Astrophys. Astron. 39, 40 (2018).

[14] L. McLerran and R. D. Pisarski, Nucl. Phys. A796, 83 (2007); Y. Hidaka, L. D. McLerran, and R. D. Pisarski, Nucl. Phys. A808, 117 (2008).

[15] L. Ya. Glozman and R. F. Wagenbrunn, Phys. Rev. D 77, 054027 (2008).
[16] D. J. Gross and A. Neveu, Phys. Rev. D 10, 3235 (1974).

[17] R. F. Dashen, B. Hasslacher, and A. Neveu, Phys. Rev. D 12, 2443 (1975).

[18] S.-S. Shei, Phys. Rev. D 14, 535 (1976).

[19] J. Feinberg and A. Zee, Phys. Rev. D 56, 5050 (1997).

[20] G. Basar and G. V. Dunne, Phys. Rev. D 78, 065022 (2008).

[21] V. Schon and M. Thies, Phys. Rev. D 62, 096002 (2000).

[22] M. Thies, J. Phys. A 39, 12707 (2006).

[23] G. Basar, G. V. Dunne, and M. Thies, Phys. Rev. D 79, 105012 (2009).

[24] G. 't Hooft, Nucl. Phys. B75, 461 (1974).

[25] G. Veneziano, Nucl. Phys. B117, 519 (1976).

[26] E. Witten, Nucl. Phys. B160, 57 (1979); B223, 433 (1983).

[27] T. H. R. Skyrme, Proc. R. Soc. A 260, 127 (1961); 262, 237 (1961); Nucl. Phys. 31, 556 (1962).

[28] N.S. Manton and P. Sutcliffe, Topological Solitons, Cambridge Monographs on Mathematical Physics (Cambridge University Press, Cambridge, England, 2004).

[29] M. Shifman and A. Yung, Supersymmetric Solitons, Cambridge Monographs on Mathematical Physics (Cambridge University Press, Cambridge, England, 2009).

[30] D. Finkelstein and J. Rubinstein, J. Math. Phys. (N.Y.) 9, 1762 (1968).

[31] A. P. Balachandran, V. P. Nair, N. Panchapakesan, and S. G. Rajeev, Phys. Rev. D 28, 2830 (1983).

[32] A. P. Balachandran, A. Barducci, F. Lizzi, V. G. J. Rodgers, and A. Stern, Phys. Rev. Lett. 52, 887 (1984); A. P. Balachandran, F. Lizzi, V. G. J. Rodgers, and A. Stern, Nucl. Phys. B256, 525 (1985).

[33] G. S. Adkins, C. R. Nappi, and E. Witten, Nucl. Phys. B228, 552 (1983). 
[34] L. Brey, H. A. Fertig, R. Cote, and A. H. MacDonald, Phys. Rev. Lett. 75, 2562 (1995).

[35] I. R. Klebanov, Nucl. Phys. B262, 133 (1985).

[36] E. Wuest, G. E. Brown, and A. D. Jackson, Nucl. Phys. A468, 450 (1987).

[37] N. S. Manton, Phys. Lett. B 192, 177 (1987).

[38] A. S. Goldhaber and N. S. Manton, Phys. Lett. B 198, 231 (1987).

[39] S. Bertini, S. L. Cacciatori, and B. L. Cerchiai, J. Math. Phys. (N.Y.) 47, 043510 (2006).

[40] S. L. Cacciatori, F. Dalla Piazza, and A. Scotti, Trans. Am. Math. Soc. 369, 4709 (2017).

[41] T. E. Tilma and G. Sudarshan, J. Geom. Phys. 52, 263 (2004).

[42] F. Canfora, Phys. Rev. D 88, 065028 (2013); Eur. Phys. J. C 78, 929 (2018).

[43] S. Chen, Y. Li, and Y. Yang, Phys. Rev. D 89, 025007 (2014).

[44] F. Canfora, M. Di Mauro, M. A. Kurkov, and A. Naddeo, Eur. Phys. J. C 75, 443 (2015).

[45] E. Ayon-Beato, F. Canfora, and J. Zanelli, Phys. Lett. B 752 , 201 (2016).

[46] P. D. Alvarez, F. Canfora, N. Dimakis, and A. Paliathanasis, Phys. Lett. B 773, 401 (2017).
[47] L. Avilés, F. Canfora, N. Dimakis, and D. Hidalgo, Phys. Rev. D 96, 125005 (2017).

[48] F. Canfora, M. Lagos, S. H. Oh, J. Oliva, and A. Vera, Phys. Rev. D 98, 085003 (2018).

[49] F. Canfora, N. Dimakis, and A. Paliathanasis, Eur. Phys. J. C 79, 139 (2019).

[50] F. Canfora, S. H. Oh, and A. Vera, Eur. Phys. J. C 79, 485 (2019).

[51] D. J. Kaup, Phys. Rev. 172, 1331 (1968).

[52] S. L. Liebling and C. Palenzuela, Living Rev. Relativity 15, 6 (2012); 20, 5 (2017).

[53] V. B. Kopeliovich, B. Schwesinger, and B. E. Stern, Pis'ma Zh. Eksp. Teor. Fiz. 62, 177 (1995) [JETP Lett. 62, 185 (1995)].

[54] Y. Brihaye, B. Hartmann, T. Ioannidou, and W. Zakrzewski, Phys. Rev. D 69, 124035 (2004).

[55] A. M. Din and W. J. Zakrzewski, Nucl. Phys. B174, 397 (1980).

[56] T. A. Ioannidou, B. Piette, and W. J. Zakrzewski, J. Math. Phys. (N.Y.) 40, 6223 (1999).

[57] P. Sutcliffe, J. High Energy Phys. 08 (2010) 019.

[58] K. Takayama and M. Oka, Nucl. Phys. A551, 637 (1993). 